\title{
Large amplitude forced vibration analysis of cross-beam system through energy method
}

\author{
A. Mitra", P. Sahoo, K. N. Saha \\ Department of Mechanical Engineering, Jadavpur University, Kolkata 700032, INDIA \\ "Corresponding Author: e-mail: samik893@gmail.com
}

\begin{abstract}
Large amplitude forced vibration behaviour of cross-beam system under harmonic excitation is studied, incorporating the effect of geometric non-linearity. The forced vibration analysis is carried out in an indirect way, in which the dynamic system is assumed to satisfy the force equilibrium condition at peak load value, thus reducing the problem to an equivalent static case. The two beams of the system are solved individually either by a direct substitution method employing successive relaxation technique or a multi-dimensional secant method, known as Broyden's method. An iterative scheme, based on the reaction force between the two beams of the system and equality of vibration amplitude at the contact of two beams, is developed to obtain the response of the system under external harmonic excitation. The present method is validated through results generated in ANSYS 11.0. The system response is studied for various loading type and contact location of the beams. The results are presented in terms of plots of vibration amplitude of the contact point versus frequency of forcing excitation in dimensional plane.
\end{abstract}

Keywords: Cross-beam, Forced vibration, Geometric non-linearity, Variational methods, Harmonic excitation

\section{Introduction}

A beam is one of the basic structural elements, which is extensively used in many branches of modern civil, mechanical, and aerospace engineering separately or in association with other beams or plates to satisfy different structural requirements like stiffness enhancement, light weight, low cost, material saving, etc. Therefore, dynamic response, especially nonlinear vibration analysis, of single beam and beam systems has always been an area of immense interest to researchers. In structural mechanics applications nonlinear system response can occur due to two reasons - material nonlinearity and geometric nonlinearity. In the first case the constituent material behaviour, i.e., stress-strain relationship is nonlinear, whereas in geometric nonlinearity the straindisplacement relationship is nonlinear. Geometric nonlinearity is caused by large amplitude transverse displacement and it induces a stretching effect, which provides additional stiffening to the structure. However, due to inclusion of the stretching effect the dynamic response becomes a nonlinear function of amplitude of excitation. The study of large displacement dynamic response of beams under external excitation has also gained tremendous attention among the modern researchers.

Research studies carried out in the field of nonlinear forced vibration analysis of beams have been chronicled in different review papers. Sathyamoorthy (1982a) reviewed the works on classical methods of non-linear (geometric, material and other type of nonlinearities) beam analysis and also surveyed the developments on non-linear beam analysis (1982b) under static and dynamic conditions using finite elements methods. Marur also (2001) put forward a review work, which reports the non-linear vibration formulations of beams through different phases of development.

One of the earlier works in this field was by Srinivasan (1966), who employed the Ritz-Galerkin technique to solve the governing nonlinear differential equation of dynamic equilibrium for forced vibration of a simply supported beam. Evenson (1968) used the perturbation method to extend the study to observe the effects of various boundary conditions. Bennet and Eisley (1970) employed a multiple mode Galerkin approach to study the nonlinear forced response of a clamped beam under concentrated harmonic force. Mei and Decha-Umphai (1985) used finite element formulation for nonlinear vibration analysis of beams subjected to harmonic excitation. Doong and Chen (1988) analysed large amplitude vibration of simply supported beams subjected 
to initial stress due to a combination of a pure bending and an extension of the neutral axis of the beam on the basis of a higherorder deformation theory. They considered shear correction factors and used Galerkin method to reduce the governing nonlinear partial differential equations to ordinary nonlinear differential equations. Leissa (1989) described an exact method for determining the vibratory displacements of an Euler-Bernoulli beam subjected to distributed excitation forces. He obtained a fourth order spatially dependent ordinary differential equation, which upon solution and application of the boundary conditions yielded a closed form solution. Chen et al (1994) devised a computational method for design sensitivity analysis of a nonlinear mode spectrum for forced vibrations of beams. Ma et al. (1995a) estimated the statistical properties of a geometrically nonlinear mode spectrum for large amplitude forced vibration systems using finite element method. The same authors (1995b) presented combined mode superposition of modal perturbation and matrix perturbation method for large deflection forced vibration of beams subjected to periodic loading incorporating both longitudinal displacement and longitudinal inertia in the formulation. Other authors have also used the perturbation method to analyze beam related problems. Maccari (1999) adopted an asymptotic perturbation method to the study of the vibrations of Euler-Bernoulli beam resting on a nonlinear elastic foundation. Ozkaya (2002) applied the perturbation method to search for a nonlinear solution to a beam-mass system made up of a simply supported Euler-Bernoulli beam carrying concentrated masses.

Fung and Chen (1997) studied the free and forced vibration of cantilever Euler-Bernoulli beam in contact with a rigid cylindrical foundation. Governing differential equations were developed through variational calculus and solution algorithm involved RungeKutta method. Fan et al (1998) proposed a method for the forced response analysis of a beam with viscoelastic boundary supports, based on complex normal mode analysis. Wang and Chou (1998) carried out nonlinear dynamic analysis of a Timoshenko beam, under the coupling effect of external moving force with the self-weight of the beam, employing large deflection theory. Azrar et al (1999) developed a semi-analytical method for analysing nonlinear dynamic response of beams using the Hamilton's principle, spectral analysis, Lagrange's equations and harmonic balance method and studied the single mode analysis for simply-supported and clamped beams. The authors (2000) extended the method to a multi mode analysis and carried out an extensive study involving various forms of excitations and boundary conditions of beams. Abu-Hilal (2003) used dynamic Green functions in determining the dynamic response of prismatic damped Euler-Bernoulli beams subjected to distributed and concentrated loads. Geometric nonlinear large amplitude forced vibration of beams was studied by Ribeiro (2004) using shooting, Newton and pversion, hierarchical finite element methods. Aristizabal-Ochoa (2004) derived undamped natural frequencies and modes of vibration of 2D Timoshenko beam-columns under constant axial load with generalized end conditions, including the combined effects of bending and shear deformations and also rotational inertia. Wu (2005) established an improved approach for obtaining classical closed form solutions for forced vibration of a beam excited by displacement boundary conditions. Das et al (2007) presented a study on geometric nonlinear large displacement forced vibration analysis of slender beams under harmonic excitation for different loading pattern and boundary conditions using energy methods and variational formulation. Sapountzakis and Dourakopoulos (2009a, 2009b) introduced a boundary element method (BEM) for nonlinear dynamic analysis of beams having double symmetric constant cross-section under general boundary conditions and subjected to a combination of transverse and axial loading. They included the effect of rotary inertia and shear deformation and employed the modified Newton-Raphson method to solve the nonlinear coupled system of equations of motion.

Forced vibration study of beam systems consisting of more than a single beam has also been a popular area of research. Ewing and Mirsafian (1996) put forward an analytical model for forced vibration of a system of two Euler-Bernoulli beams joined with a nonlinear rotational torsional spring with linear and cubic stiffness. The authors used harmonic balance method to find an approximate solution for beams with simply supported and clamped end conditions. Oniszczuk (2003) applied the classical modal expansion method for analysing undamped forced transverse vibrations of an elastically connected complex simply-supported double-beam system. The stability and free vibration analyses of a Timoshenko beam-column with generalized end conditions subjected to constant axial load, and weakened by a cracked section along its span was presented by Arboleda-Monsalve et al (2007). Modeling of the crack as an intermediate flexural connection of zero length produced a system with two-segments having rotational discontinuity at the crack location but identical lateral deflection.

The present paper undertakes a large displacement geometric nonlinear forced vibration analysis of a two beam system under harmonic excitation. The system comprises of two beams perpendicular to each other and placed in contact, one over the other. Such cross-beam systems are quite common in civil, mechanical and construction engineering applications. The dynamic system is assumed to satisfy the force equilibrium condition at peak excitation amplitude. This assumption reduces the problem to an equivalent static analysis, where the geometric non-linearity is taken into account by considering the non-linear straindisplacement relations and an energy approach is employed to arrive at the appropriate governing equations. The formulation is displacement based and the unknown displacement fields are approximated by finite linear combination of admissible orthogonal functions. Unlike the FEM, where the physical domain is discretised into smaller finite elements, the present semi-analytical formulation is associated with the whole domain i.e. the assumed displacement field is a function of the whole physical domain. As a result, the number of algebraic equations to be solved is substantially reduced compared to FEM. A direct substitution method with relaxation parameter is used to solve the set of non-linear equations. Also a multidimensional quasi-Newton method known as Broyden method is employed for solution purpose. The displacement fields are solved through an iterative procedure which is based on the reaction force between the two beams of the system and considers the difference between the displacements of the 
two beams at their contact point as the convergence criterion. Results are presented in graphical form through frequency-amplitude plots in dimensional plane.

\section{Analysis}

The beam system, analyzed in the present paper, consists of two slender beams in contact with their longitudinal axes perpendicular to each other so as to form a 'cross', as shown in Figure 1. The beams are in surface contact and at no loading condition there is no interaction between them. But when some transverse load is applied on the upper beam (Beam-1), it pushes down on the lower beam (Beam-2), which in turn provides a resistance to deformation by sharing a portion of the load. It is assumed that external transverse harmonic excitation acts on beam-1 only and the purpose of beam-2 is to give the system a stiffening effect. It is also implied that the deformation amplitude at the point of contact of the two beams are equal i.e. there is no detachment of the contact point at any instant.

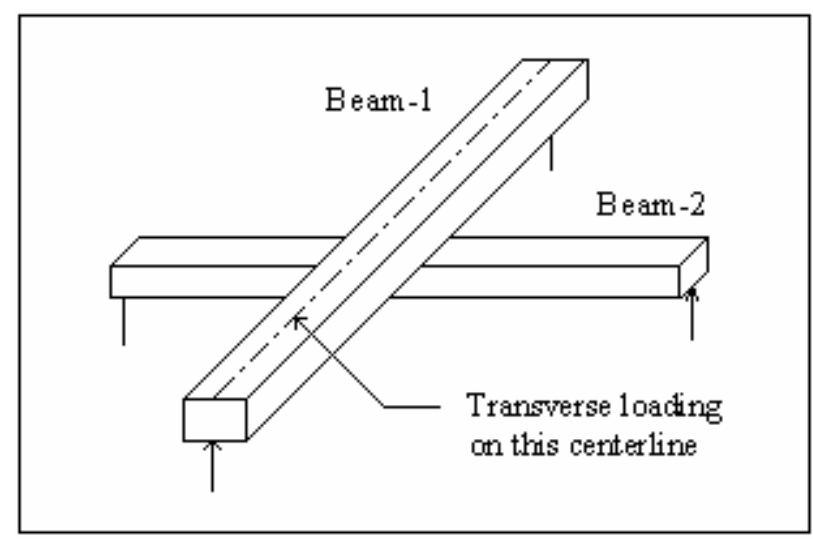

Figure 1. Cross-beam system

Figure 2 shows the constituent beams of the system under external loading at a certain time instant with the indications of nomenclatures of some system parameters. In this figure, $R$ represents the unique reaction force generated at the contact between two beams. In the present formulation the beam system is modeled as two individual beams, which incorporate the reaction force $(R)$ as an external force describing the effect of the other beam. It is also assumed that the reaction force $(R)$ has the same frequency $(\omega)$ as the transverse harmonic excitation and there is no lead or lag between the two. Actually, the contact between the two beams is over a patch of area where a distributed reaction force would act. In order to simplify the situation it is assumed that the contact between the two beams occurs at a point and the connection is such that only vertical forces can be transmitted through it. This is an idealization of a general situation where reaction forces can be taken to act in all three directions.
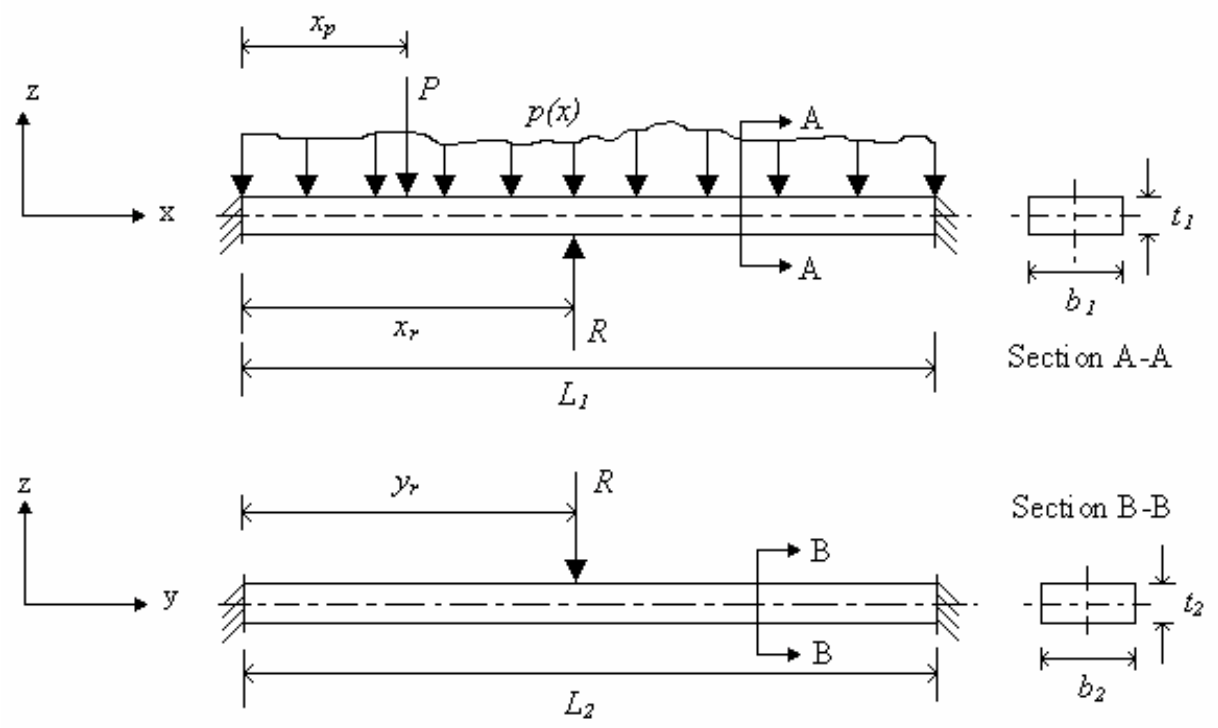

Figure 2. Individual beams with external loading and reaction force at the contact point of two beams. 
In the present study forced vibration analyses of cross-beams are carried out numerically. The system response is shown in displacement amplitude vs. frequency of external harmonic excitation plane, while the amplitude of external excitation is kept fixed. A family of displacement amplitude-excitation frequency curves can be generated for different amplitude of external excitation. This family of curves for different excitation values indicates functional relationship of the displacement of cross-beam with the frequency of excitation and is known as the frequency response of the cross-beam. It is to be mentioned that the present study is limited to only the steady state dynamic response of the system under harmonic transverse loading. The mathematical formulation is energy based and the set of governing equations is derived using Hamilton's principle. The solution is approximate in nature due to the assumption of the individual dynamic displacement fields as finite linear combinations of orthogonal admissible functions and unknown coefficients. The set of non-linear equations for forced vibration problem is solved by applying direct substitution technique with successive relaxation scheme. Also Broyden's method, which is a globally convergent solution algorithm, is employed separately as it is found to be successful over a wider range of system parameters.

The formulation is generalized in nature to analyze problems for any classical boundary conditions and loading pattern. Although the formulation can handle any classical boundary conditions (for example, clamped, simply supported and free), in the present paper only clamped boundary conditions at all the ends is considered. However, four different types of loadings namely, concentrated load, uniformly distributed load (UDL), triangular load and hat type load are considered. Figure 3(1-4) depicts schematic diagrams of loading patterns on beam-1 with clamped end conditions.

\subsection{Mathematical formulation}

In the present study forced vibration analysis is carried out in an indirect way. The external excitation is considered as harmonic and the system response is assumed to have the same frequency as that of the external excitation. The basis of this assumption is that a harmonic load is going to produce a harmonic response amplitude of the same frequency (Ewing and Mirsafian 1996). To determine the frequency response of the cross-beam a frequency sweep is carried out at a particular load level of the external excitation. The sweep is started at zero value of the excitation frequency and steadily increased beyond resonance till the response amplitude falls to a sufficiently low level. Corresponding to each frequency of that particular load level, the dynamic response is made to maintain force equilibrium condition at the peak load level. Thus the dynamic problem is solved as a static one, whose response, quite obviously, is a function of the external excitation frequency and amplitude of loading. After the responses of the two beams under their respective loadings are obtained, the displacement amplitude at the contact point for the two beams is compared. The details of the solution procedure are elaborated in a later section.

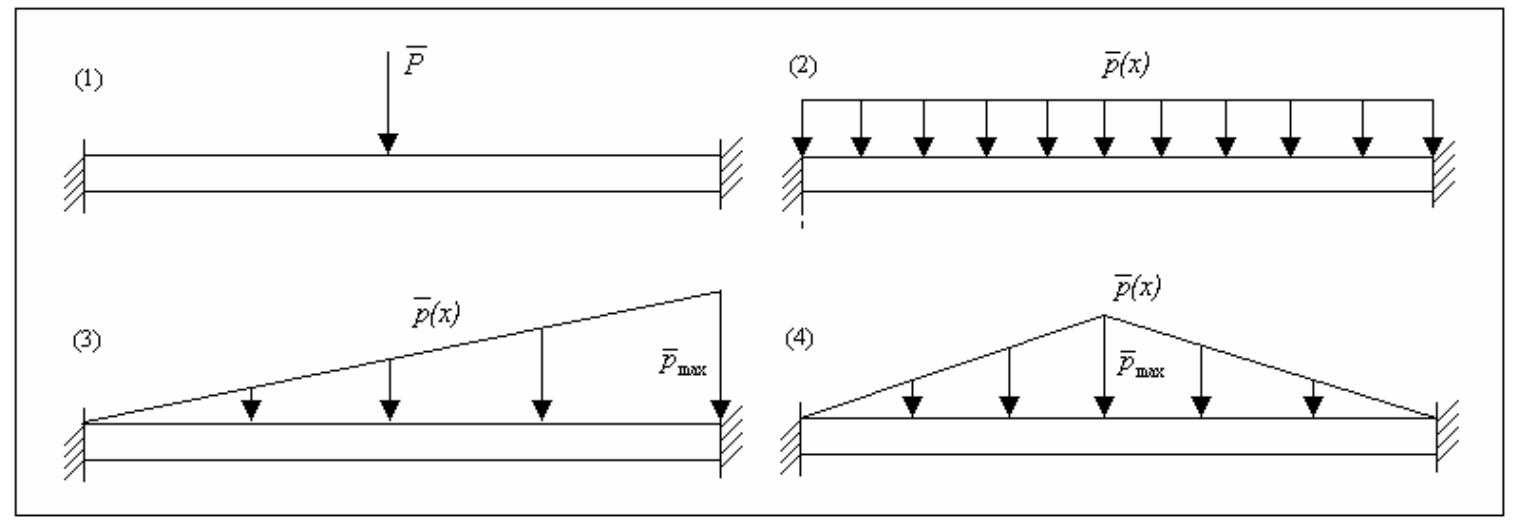

Figure 3. Types of loading applied on beam-1: 1) Concentrated, 2) Uniformly distributed, 3) Triangular and 4) Hat

The mathematical formulation of the individual beams is based on the following assumptions -

1) Beam materials are isotropic, homogeneous and linearly elastic.

2) Beams follow Euler - Bernoulli hypothesis i.e. the thicknesses of the beams are small compared to their respective lengths, such that the effect of shear deformation and rotary inertia may be neglected

3) Beams have uniform rectangular cross-section.

The governing set of equations for the dynamic system is obtained applying the Hamilton's principle (Mierovitch 1970), which is given by,

$$
\delta\left(\int_{\tau_{1}}^{\tau_{2}}(T-U-V) d \tau\right)=\delta\left(\int_{\tau_{1}}^{\tau_{2}}(T-\pi) d \tau\right)=\delta\left(\int_{\tau_{1}}^{\tau_{2}} L d \tau\right)=0
$$


where, $T, U$ and $V$ are the total kinetic energy of the system, total strain energy stored in the system and work function or potential of the external forces respectively. $L, \delta$ and $\tau$ represent the Lagrangian, the variational operator and time coordinate respectively. The above mentioned energy functionals are determined from the assumed dynamic displacement fields.

In case of large displacement analysis of beams, both bending and stretching effects are taken into consideration. Bending action is caused by transverse load and contributes to transverse displacement, whereas stretching displacement is caused by in-plane restraints to displacement. So, total strain energy stored in beam-1 is given by,

$$
U_{1}=U_{b 1}+U_{m 1}
$$

where, $U_{b 1}=$ Strain energy stored due to bending $=\frac{E_{1}}{2} \int_{v o l}\left(\varepsilon_{x}^{b}\right)^{2} d v$

$$
U_{m 1}=\text { Strain energy stored due to stretching }=\frac{E_{1}}{2} \int_{v o l}\left(\varepsilon_{x}^{s}\right)^{2} d v
$$

$\varepsilon_{x}^{b}$ and $\varepsilon_{x}^{s}$ are axial strains due to bending and stretching respectively. The axial strain due to bending at a distance $Z$ from the mid-plane is given by, $\varepsilon_{x}^{b}=-z \frac{d^{2} w_{1}}{d x^{2}}$ and axial strain due to stretching of mid-plane is given by, $\varepsilon_{x}^{s}=\frac{d u_{1}}{d x}+\frac{1}{2}\left(\frac{d w_{1}}{d x}\right)^{2}$. Substituting these strain expressions into equation (2), the total strain energy stored in beam- 1 is,

$$
U_{1}=\frac{E_{1} I_{1}}{2} \int_{0}^{L_{1}}\left(\frac{d^{2} w_{1}}{d x^{2}}\right)^{2} d x+\frac{E_{1} A_{1}}{2} \int_{0}^{L_{1}}\left[\left(\frac{d u_{1}}{d x}\right)^{2}+\frac{1}{4}\left(\frac{d w_{1}}{d x}\right)^{4}+\left(\frac{d u_{1}}{d x}\right) \cdot\left(\frac{d w_{1}}{d x}\right)^{2}\right] d x
$$

Similarly, the total strain energy stored in beam-2 is given by,

$$
U_{2}=\frac{E_{2} I_{2}}{2} \int_{0}^{L_{2}}\left(\frac{d^{2} w_{2}}{d y^{2}}\right)^{2} d y+\frac{E_{2} A_{2}}{2} \int_{0}^{L_{2}}\left[\left(\frac{d u_{2}}{d y}\right)^{2}+\frac{1}{4}\left(\frac{d w_{2}}{d y}\right)^{4}+\left(\frac{d u_{2}}{d y}\right) \cdot\left(\frac{d w_{2}}{d y}\right)^{2}\right] d y
$$

where, $w_{1}, w_{2}$ are transverse dynamic displacements of mid-plane of beam- 1 and beam- 2 respectively, $u_{1}, u_{2}$ are in-plane dynamic displacements of mid-plane of beam- 1 and beam- 2 respectively, $E_{1}, E_{2}$ are elastic modulii of beam- 1 and beam- 2 respectively, $L_{1}, L_{2}$ are lengths of beam- 1 and beam-2 respectively, $x, y$ are axial coordinates in two orthogonal directions. $I_{1}, I_{2}$ and $A_{1}, A_{2}$ are second moment of area and cross sectional area of the two beams respectively. The computations are carried out in normalized coordinates $\xi$ and $\eta$ respectively, where $\xi=\frac{x}{L_{1}}$ and $\eta=\frac{y}{L_{2}}$. In arriving at equation (3) \& (4), the following properties of beam cross-section have been utilized:

$$
b_{i} \int_{-t_{i} / 2}^{t_{i} / 2} d z=A_{i}, b_{i} \int_{-t_{i} / 2}^{t_{i} / 2} z d z=0, b_{i} \int_{-t_{i} / 2}^{t_{i} / 2} z^{2} d z=I_{i}, b_{i} \int_{-t_{i} / 2}^{t_{i} / 2} z^{3} d z=0 \quad i=1,2
$$

As the frequency of response is considered identical with the excitation frequency, determination of the kinetic energy functional becomes straightforward. The expression for total kinetic energy of beam- 1 and beam- 2 are given by,

$$
\begin{aligned}
& T_{1}=\frac{1}{2} \rho_{1} b_{1} t_{1} \int_{0}^{L_{1}}\left[\left(\frac{\partial w_{1}}{\partial \tau}\right)^{2}+\left(\frac{\partial u_{1}}{\partial \tau}\right)^{2}\right] d x \\
& T_{2}=\frac{1}{2} \rho_{2} b_{2} t_{2} \int_{0}^{L_{2}}\left[\left(\frac{\partial w_{2}}{\partial \tau}\right)^{2}+\left(\frac{\partial u_{2}}{\partial \tau}\right)^{2}\right] d y
\end{aligned}
$$

where, $\rho_{1}, \rho_{2}$ are densities of beam- 1 and beam- 2 materials, respectively.

The work potential of the external load for beam- 1 and beam- 2 are computed corresponding to peak load level and given by,

$$
\begin{aligned}
& V_{1}=\left.P w_{1}\right|_{x_{p}}+\int_{0}^{L_{1}}\left(p w_{1}\right) d x-\left.R w_{1}\right|_{x_{r}} \\
& V_{2}=\left.R w_{2}\right|_{y_{r}}
\end{aligned}
$$


where, $P$ and $p$ represent harmonic excitation for concentrated and pressure type loading, whose expressions are given by $P=\bar{P} e^{i \omega \tau}$ and $p=\bar{p}(x) e^{i \omega \tau}$, where, $\omega$ denotes the frequency of excitation and $i=\sqrt{-1} . \bar{P}$ represent the amplitude of harmonic excitation for concentrated loading and $\bar{p}$ represents the intensity of harmonic excitation per unit length of the beam for pressure type loading. It should be mentioned that $\bar{p}_{\max }$ is the amplitude of maximum intensity for triangular and hat type loadings. The loading is acting on beam-1 and $R$ is the reaction force generated between the two beams and has the same frequency and phase as the external harmonic excitation as mentioned earlier. The expression for the reaction force is given as $R=\bar{R} e^{i \omega \tau}$, where $\bar{R}$ denotes the amplitude of the reaction. The coordinate, $x_{p}$ represents the point of application of the external concentrated harmonic excitation on beam- 1 and $x_{r}, y_{r}$ denote the location, where the reaction force $(R)$ acts on beam- 1 and beam-2 respectively. In other words, $x_{r}$ and $y_{r}$ describe the contact point between the two beams. These notations have been indicated in Figure 2 also.

The dynamic displacements $w_{1}(\xi, \tau), w_{2}(\xi, \tau)$ and $u_{1}(\xi, \tau), u_{2}(\xi, \tau)$ are assumed to be separable in space and time (Fan et al 1998; Kadiri et al 2002) and can be approximately represented by sets of orthogonal coordinate functions $\phi_{1 i}, \phi_{2 i}$ and $\alpha_{1 i}, \alpha_{2 i}$.

$$
\begin{aligned}
& w_{1}(\xi, \tau)=\sum_{i=1}^{n w} d_{1 i} \phi_{1 i}(\xi) e^{i \omega \tau} \text { and } u_{1}(\xi, \tau)=\sum_{i=n w+1}^{n w+n u} d_{1 i} \alpha_{1 i-n w}(\xi) e^{i \omega \tau} \\
& w_{2}(\eta, \tau)=\sum_{i=1}^{n w} d_{2 i} \phi_{2 i}(\eta) e^{i \omega \tau} \text { and } u_{2}(\eta, \tau)=\sum_{i=n w+1}^{n w+n u} d_{2 i} \alpha_{2 i-n w}(\eta) e^{i \omega \tau}
\end{aligned}
$$

where, $d_{1 i}$ and $d_{2 i}$ represent unknown coefficients and $n w$ and $n u$ are number of functions for $w$ and $u$ respectively. Appropriate start functions for $w_{1}, u_{1}$ and $w_{2}, u_{2}$ are selected in such a way that they satisfy the necessary geometric boundary conditions of the respective beams. The higher order functions are generated from the selected start functions using Gram-Schmidt orthogonalisation scheme.

Substituting equations (3), (5), (7), (9) and (4), (6), (8), (10) separately in equation (1) gives the governing set of equations for beam- 1 and beam- 2 respectively. For beam-1, the governing set of equations in matrix form is given by,

$$
\left[K^{1}\right]\left\{d_{1}\right\}-\omega^{2}\left[M^{1}\right]\left\{d_{1}\right\}=\left\{f_{1}\right\}
$$

where, $\left[K^{1}\right],\left[M^{1}\right]$ and $\left\{f_{1}\right\}$ are stiffness matrix, mass matrix and load vector respectively and are of the following form.

$$
\left[K^{1}\right]=\left[\begin{array}{ll}
K_{11}^{1} & K_{12}^{1} \\
K_{21}^{1} & K_{22}^{1}
\end{array}\right],\left[M^{1}\right]=\left[\begin{array}{ll}
M_{11}^{1} & M_{12}^{1} \\
M_{21}^{1} & M_{22}^{1}
\end{array}\right] \text { and }\left\{f_{1}\right\}=\left\{\begin{array}{c}
f_{11}^{1} \\
f_{12}^{1}
\end{array}\right\} \text {. }
$$

The elements of $\left[K^{1}\right],\left[M^{1}\right]$ and $\left\{f_{1}\right\}$ are:

$$
\begin{aligned}
{\left[K_{11}^{1}\right] } & =\frac{E_{1} I_{1}}{L_{1}^{3}} \sum_{j=1}^{n w} \sum_{i=1}^{n w} \int_{0}^{1} \frac{d^{2} \phi_{1 i}}{d \xi^{2}} \frac{d^{2} \phi_{1 j}}{d \xi^{2}} d \xi+\frac{E_{1} A_{1}}{2 L_{1}^{3}} \sum_{j=1}^{n w} \sum_{i=1}^{n w} \int_{0}^{1}\left(\sum_{i=1}^{n w} d_{1 i} \frac{d \phi_{1 i}}{d \xi}\right)^{2} \frac{d \phi_{1 i}}{d \xi} \frac{d \phi_{1 j}}{d \xi} d \xi \\
& +\frac{E_{1} A_{1}}{L_{1}^{2}} \sum_{j=1}^{n w} \sum_{i=1}^{n w} \int_{0}^{1}\left(\sum_{i=n w+1}^{n w+n u} d_{1 i} \frac{d \alpha_{1 i-n w}}{d \xi}\right)^{2} \frac{d \phi_{1 i}}{d \xi} \frac{d \phi_{1 j}}{d \xi} d \xi, \\
{\left[K_{12}^{1}\right]=} & 0,\left[K_{21}^{1}\right]=\frac{E_{1} A_{1}}{2 L_{1}^{2}} \sum_{j=n w+1}^{n w+n u} \sum_{i=1}^{n w} \int_{0}^{1}\left(\sum_{i=1}^{n w} d_{1 i} \frac{d \phi_{1 i}}{d \xi}\right) \frac{d \phi_{1 i}}{d \xi} \frac{d \alpha_{1 j}-n w}{d \xi} d \xi,\left[K_{22}^{1}\right]=\frac{E_{1} A_{1}}{L_{1}} \sum_{j=n w+1}^{n w+n u} \sum_{i=n w+1}^{n w+n u} \frac{1}{d} \frac{d \alpha_{1 i-n w}}{d \xi} \frac{d \alpha_{1 j-n w}}{d \xi} d \xi, \\
{\left[M_{11}^{1}\right]=} & \rho_{1} b_{1} t_{1} L_{1} \sum_{j=1}^{n w} \sum_{i=1}^{n w} \int_{0}^{1} \phi_{1 i} \phi_{1 j} d \xi,\left[M_{12}^{1}\right]=0,\left[M_{21}^{1}\right]=0,\left[M_{22}^{1}\right]=\rho_{1} b_{1} t_{1} L_{1} \sum_{j=n w+1}^{n w+n u} \sum_{i=n w+1}^{n w+n u} \int_{0}^{1} \alpha_{1 i-n w} \alpha_{1 j-n w} d \xi \\
\left\{f_{11}^{1}\right\}= & \sum_{j=1}^{n w} \bar{P} \phi_{1 j}\left|\xi_{p}+L_{1} \sum_{j=1}^{n w} \int_{0}^{1} \bar{p}(\xi) \phi_{1 j} d \xi-\sum_{j=1}^{n w} \bar{R} \phi_{1 j}\right|_{\xi_{r}},\left\{f_{12}^{1}\right\}=0,
\end{aligned}
$$

where, $\xi_{p}$ and $\xi_{r}$ represent the point of application of the external concentrated harmonic excitation and the location, where the reaction force $(R)$ acts on beam- 1 in the normalized scale. It is seen that some of the terms of stiffness matrix ([ $\left.K_{11}^{1}\right]$ and [ $\left.K_{21}^{1}\right]$ ) 
contain the undetermined coefficients $\left(\left\{d_{1 i}\right\}\right)$ and hence give rise to nonlinearity. So, these specific terms can be called as nonlinear terms.

Similarly, for beam-2, the governing set of equations in matrix form is given by,

$$
\left[K^{2}\right]\left\{d_{2}\right\}-\omega^{2}\left[M^{2}\right]\left\{d_{2}\right\}=\left\{f_{2}\right\}
$$

where, $\left[K^{2}\right],\left[M^{2}\right]$ and $\left\{f_{2}\right\}$ are stiffness matrix, mass matrix and load vector respectively and are of the following form.

$$
\left[K^{2}\right]=\left[\begin{array}{ll}
K_{11}^{2} & K_{12}^{2} \\
K_{21}^{2} & K_{22}^{2}
\end{array}\right],\left[M^{2}\right]=\left[\begin{array}{ll}
M_{11}^{2} & M_{12}^{2} \\
M_{21}^{2} & M_{22}^{2}
\end{array}\right] \text { and }\left\{f_{2}\right\}=\left\{\begin{array}{c}
f_{11}^{2} \\
f_{12}^{2}
\end{array}\right\} \text {. }
$$

The elements of $\left[K^{2}\right],\left[M^{2}\right]$ and $\left\{f_{2}\right\}$ are:

$$
\begin{aligned}
{\left[K_{11}^{2}\right] } & =\frac{E_{2} I_{2}}{L_{2}^{3}} \sum_{j=1}^{n w} \sum_{i=1}^{n w} \int_{0}^{1} \frac{d^{2} \phi_{2 i}}{d \eta^{2}} \frac{d^{2} \phi_{2 j}}{d \eta^{2}} d \eta+\frac{E_{2} A_{2}}{2 L_{2}^{3}} \sum_{j=1}^{n w} \sum_{i=1}^{n w} \int_{0}^{1}\left(\sum_{i=1}^{n w} d_{2 i} \frac{d \phi_{2 i}}{d \eta}\right)^{2} \frac{d \phi_{2 i}}{d \eta} \frac{d \phi_{2 j}}{d \eta} d \eta \\
& +\frac{E_{2} A_{2}}{L_{2}^{2}} \sum_{j=1}^{n w} \sum_{i=1}^{n w} \int_{0}^{1}\left(\sum_{i=n w+1}^{n w+n u} d_{2 i} \frac{d \alpha_{2 i-n w}}{d \eta}\right)^{2} \frac{d \phi_{2 i}}{d \eta} \frac{d \phi_{2 j}}{d \eta} d \eta, \\
{\left[K_{12}^{2}\right]=} & 0,\left[K_{21}^{2}\right]=\frac{E_{2} A_{2}}{2 L_{2}^{2}} \sum_{j=n w+1}^{n w+n u} \sum_{i=1}^{n w} \int_{0}^{1}\left(\sum_{i=1}^{n w} d_{2 i} \frac{d \phi_{2 i}}{d \eta}\right) \frac{d \phi_{2 i}}{d \eta} \frac{d \alpha_{2 j-n w}}{d \eta} d \eta,\left[K_{22}^{2}\right]=\frac{E_{2} A_{2}}{L_{2}} \sum_{j=n w+1 i=n w+1}^{n w+n u} \sum_{0}^{n w+n u} \frac{1}{d \eta} \frac{d \alpha_{2 i-n w}}{d \eta} \frac{d \alpha_{2 j}-n w}{d \eta} d \eta, \\
{\left[M_{11}^{2}\right]=} & \rho_{2} b_{2} t_{2} L_{2} \sum_{j=1}^{n w} \sum_{i=1}^{n w} \int_{0}^{1} \phi_{2 i} \phi_{2 j} d \eta,\left[M_{12}^{2}\right]=0,\left[M_{21}^{2}\right]=0,\left[M_{22}^{2}\right]=\rho_{2} b_{2} t_{2} L_{2} \sum_{j=n w+1}^{n w+n u} \sum_{i=n w+1}^{n w+n u} \int_{0}^{1} \alpha_{2 i-n w} \alpha_{2 j-n w} d \eta \\
\left\{f_{11}^{2}\right\}= & \left.\sum_{j=1}^{n w} \bar{R} \phi_{2 j}\right|_{\eta_{r}},\left\{f_{12}^{2}\right\}=0,
\end{aligned}
$$

where, $\eta_{r}$ is the normalized axial coordinate of the location, where the reaction force $(R)$ acts on beam-2. Similar to the previous equation here also nonlinear terms are present in the stiffness sub-matrices [ $\left.K_{11}^{2}\right]$ and [ $K_{21}^{2}$ ].

\subsection{Solution Procedure}

Equations (11) and (12) represent the governing set of equations for the cross-beam system and are undoubtedly dynamic in nature. But solution procedure undertaken in the present scenario reduces the problem to an equivalent static case, where, the maximum amplitude of the harmonic excitation and the frequency of excitation are supplied and the corresponding undetermined coefficients are calculated. At the maximum load value it is assumed that the system satisfies the force equilibrium condition, i.e., the sum of elastic and inertia forces equal the externally applied peak load value. Thus the dynamic problem is solved as a static one where the system response becomes dependent on the excitation frequency and the maximum amplitude of the harmonic excitation. In the present paper two methods are utilized to obtain the solution.

Substitution Method: The set of governing equations (eqn. (11) and (12)) are nonlinear in nature as the stiffness matrices ([ $\left.K^{1}\right]$ and $\left.\left[K^{2}\right]\right)$ are a function of the unknown parameters and hence cannot be solved directly. They are solved through an iterative procedure utilizing the direct substitution technique with successive relaxation scheme. For the maximum amplitude and certain frequency of harmonic excitation the values of the unknown coefficients are assumed and based on that, new values of unknown coefficients are calculated by matrix inversion technique from the expressions (13a) and (13b).

$$
\begin{aligned}
& \left\{d_{1}\right\}=\left[\left[K^{1}\right]-\omega^{2}\left[M^{1}\right]\right]^{-1}\left\{f_{1}\right\} \\
& \left\{d_{2}\right\}=\left[\left[K^{2}\right]-\omega^{2}\left[M^{2}\right]\right]^{-1}\left\{f_{2}\right\}
\end{aligned}
$$

Calculated values are compared with the corresponding values in the previous iteration and if the difference is above a predefined error limit $\left(\varepsilon_{1}\right)$, the process is repeated with new values of unknown coefficients, modified with a relaxation parameter, until convergence is achieved. It can be said that the matrix $\left[K^{i}-\omega^{2} M^{i}\right], i=1,2$, is an equivalent stiffness matrix, which represents the dynamic system stiffness and it depends on the frequency of loading. Thus, from the values of $\left\{d_{1}\right\}$ and $\left\{d_{2}\right\}$, the amplitude of forced vibration can be determined in the present case instead of the static deflection. It is also important to note that with increase in the frequency of excitation, the dynamic stiffness $\left(\left[K^{i}-\omega^{2} M^{i}\right], i=1,2\right)$ may change its sign and usually it 
occurs when $\omega>\omega_{1}$, where, $\omega_{1}$ is the first natural frequency of the corresponding beam. This method is computationally efficient and requires less processing time.

Broyden's method: Broyden's method is a multidimensional quasi-Newton method for the numerical solution of a system of nonlinear equations. In this method an initial Jacobian of the system is calculated depending on the initial guess value and in the successive iterations an updated value of the Jacobian is used. Similar to the substitution scheme, in this method also values of the unknown coefficient $\left\{d_{1}\right\}$ and $\left\{d_{2}\right\}$ are solved from the governing equations shown in (14a) and (14b).

$$
\begin{aligned}
& {\left[\left[K^{1}\right]-\omega^{2}\left[M^{1}\right]\right]\left\{d_{1}\right\}-\left\{f_{1}\right\}=0} \\
& {\left[\left[K^{2}\right]-\omega^{2}\left[M^{2}\right]\right]\left\{d_{2}\right\}-\left\{f_{2}\right\}=0}
\end{aligned}
$$

The load vectors in eqn. (13) and (14) contain magnitude of loading ( $\bar{P}$ or $\bar{p})$ and reaction force $(\bar{R})$ generated. So, the major obstacle in implementing the discussed schemes is that the load vectors $\left(\left\{f_{1}\right\}\right.$ and $\left\{f_{2}\right\}$ ) are not completely known as for a particular amplitude and certain frequency of harmonic excitation applied to the cross-beam structure the reaction force $(R)$ generated at the contact between two beams is not known beforehand. To overcome this amplitude the reaction force $(\bar{R})$ is assumed to be a fraction $(q)$ of the amplitude of harmonic excitation applied to the structure. At the start, the correct value of $q$ ( $q_{\text {exact }}$ ) is unknown but the minimum and maximum values of $q$ are known to lie between 0 and 1, i.e., $q_{\min }=0$ and $q_{\max }=1$. Using this assumed value, the load vectors are calculated and the set of unknown coefficients, as well as the forced vibration amplitude values of the two beams are solved. Let the vibration amplitude of beam-1 and beam-2 at the point of contact be $\delta_{b 1}$ and $\delta_{b 2}$, respectively. If $\left(\delta_{b 1}-\delta_{b 2}\right) / \delta_{b 1}$ is outside a permitted tolerance limit $\left(\varepsilon_{2}\right)$, the assumed reaction force is changed by modifying the fraction $(q)$ and using this new assumed reaction force the process is repeated. The modification of the assumed reaction force is carried out as follows:

At the end of each iteration step, a part of the solution space can be eliminated depending on whether $\delta_{b 1}$ is greater or lower than $\delta_{b 2}$, thus changing $q_{\min }$ or $q_{\max }$.

If $\delta_{b 1}>\delta_{b 2}: q$ needs to be increased in order to lower $\delta_{b 1}$ and increase $\delta_{b 2}$. This implies that $q_{\text {exact }}$ cannot be lower than the present $q$ and $q_{\text {min }}$ can be upgraded to this $q$ value. After the modification of the limit, the new value $q$ is determined using the following expression, $q=q_{\min }+q_{1}\left(q_{\max }-q_{\min }\right)$, where $q_{1}$ is another load distribution parameter whose value lies between 0 and $1\left(0<q_{1}<1\right)$.

If $\delta_{b 1}<\delta_{b 2}: q$ needs to be decreased in order to increase $\delta_{b 1}$ and lower $\delta_{b 2}$. This implies that $q_{\text {exact }}$ cannot be greater than the present $q$ and $q_{\max }$ can be upgraded to this $q$ value. After the modification of the limit, the new value $q$ is determined using the following expression, $q=q_{\max }-q_{1}\left(q_{\max }-q_{\min }\right)$, where $0<q_{1}<1$.

When $\left(\delta_{b 1}-\delta_{b 2}\right) / \delta_{b 1}$ falls within the predetermined permissible value $\left(\varepsilon_{2}\right)$, the process is said to have converged. The solution procedure for a given set of input values is elaborated in the flow chart shown in Figure 4. 


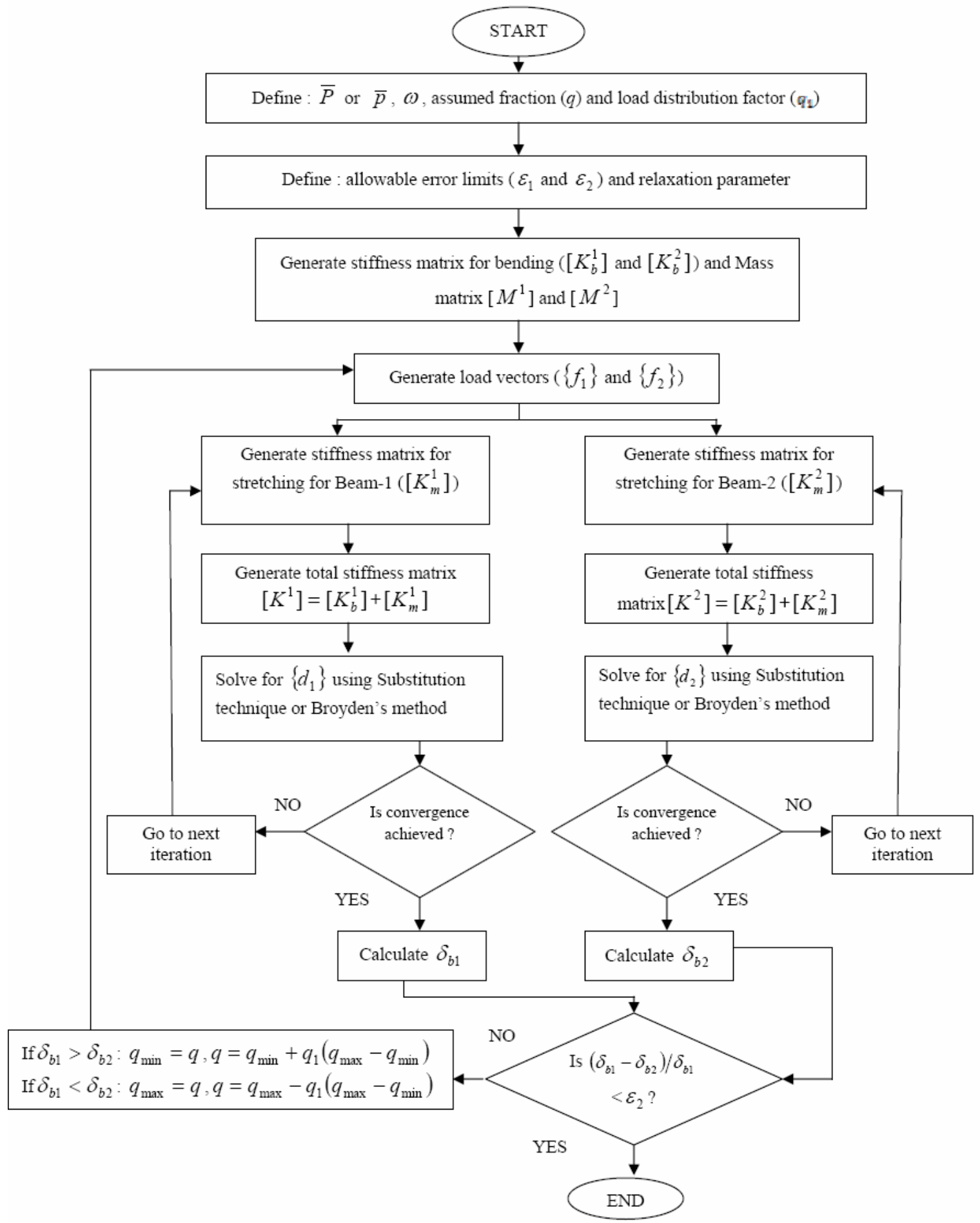

Figure 4. Flow chart of the solution algorithm for a given set of input values

\section{Results and Discussion}

The effects of loading pattern, beam geometry and variation in thickness of beam-2 on the forced vibration behaviour of the system have been studied. The results are presented through plots of vibration amplitude of the contact point versus frequency of forcing excitation in dimensional plane. In the present paper four different loading patterns, concentrated load, uniformly distributed load, triangular load and hat load are considered as shown in Figure 3. However, for all these types of loading, flexural boundary conditions of the beams have been considered as clamped. In the present study external axial loading (compression and tension) is not considered, but axial forces arise due to stretching of the beam. It is assumed that the clamped boundaries are 
sufficiently rigid to support these axial loads generated in the beams and hence, for membrane boundary conditions the in-plane displacements are taken as zero at the boundaries, i.e., $u_{1}(\xi, \tau)=0$ at $\xi=0,1$ and $u_{2}(\eta, \tau)=0$ at $\eta=0$, 1 . In all the cases, the contact point is assumed at the mid-span of beam-2, i.e., $y_{r}=0.5 L_{2}$.

The start functions for $w_{1}, u_{1}$ and $w_{2}, u_{2}$ are selected to satisfy the flexural and in-plane boundary conditions of the beams. The start functions for the beam deflection $\left(w_{1}, w_{2}\right)$ come from the flexural boundary conditions and the start functions for the stretching of the beam $\left(u_{1}, u_{2}\right)$ come from the membrane boundary conditions. Both sets of start functions are shown in Table 1 . These selected start functions are used to generate the higher order functions with the help of Gram-Schmidt orthogonalisation principle. The number of functions ( $n w$ and $n u$ ) is taken as 8 and this particular number has been arrived at after carrying out the necessary convergence tests.

Table 1. Start functions for $w_{1}, u_{1}$ and $w_{2}, u_{2}$

\begin{tabular}{|c|c|c|}
\hline \multirow{2}{*}{ Beam-1 } & $\phi_{11}(\xi)$ & $\{\xi(1-\xi)\}^{2}$ \\
\cline { 2 - 3 } & $\alpha_{11}(\xi)$ & $\xi(1-\xi)$ \\
\hline \multirow{2}{*}{ Beam-2 } & $\phi_{21}(\eta)$ & $\{\eta(1-\eta)\}^{2}$ \\
\cline { 2 - 3 } & $\alpha_{21}(\eta)$ & $\eta(1-\eta)$ \\
\hline
\end{tabular}

The solution methodology involves an iterative numerical scheme with successive relaxation and Broyden's method for solution of individual beams and a specially developed iterative procedure, based on the reaction force generated and equality of amplitude of vibration at the contact of two beams, to obtain the solution for the system under a particular load level and excitation frequency. It is observed that the convergence of the iteration scheme for the system depends on the permissible value $\left(\varepsilon_{2}\right)$ of the error limit. A convergence study is carried out on a cross-beam system having the following specifications and under uniformly distributed loading intensity of $100 \mathrm{~N} / \mathrm{m}$ - Dimensions $L_{1}=1 \mathrm{~m}, b_{1}=0.02 \mathrm{~m}, t_{1}=0.01 \mathrm{~m}$ and $L_{2}=1 \mathrm{~m}, b_{2}=0.02 \mathrm{~m}, t_{2}=0.01 \mathrm{~m}$, Material property: $E_{1}=E_{2}=210 \mathrm{GPa}, \rho_{1}=\rho_{2}=7850 \mathrm{~kg} / \mathrm{m}^{3}$, Contact Locations : $x_{r}=0.5 L_{1}$ and $y_{r}=0.5 L_{2}$. Figure 5 shows the convergence results from the above mentioned study at two values $(\omega=0,15.91 \mathrm{~Hz}$.) of the external excitation frequency and indicates that $0.01 \%$ can be taken as an acceptable error limit for the study.

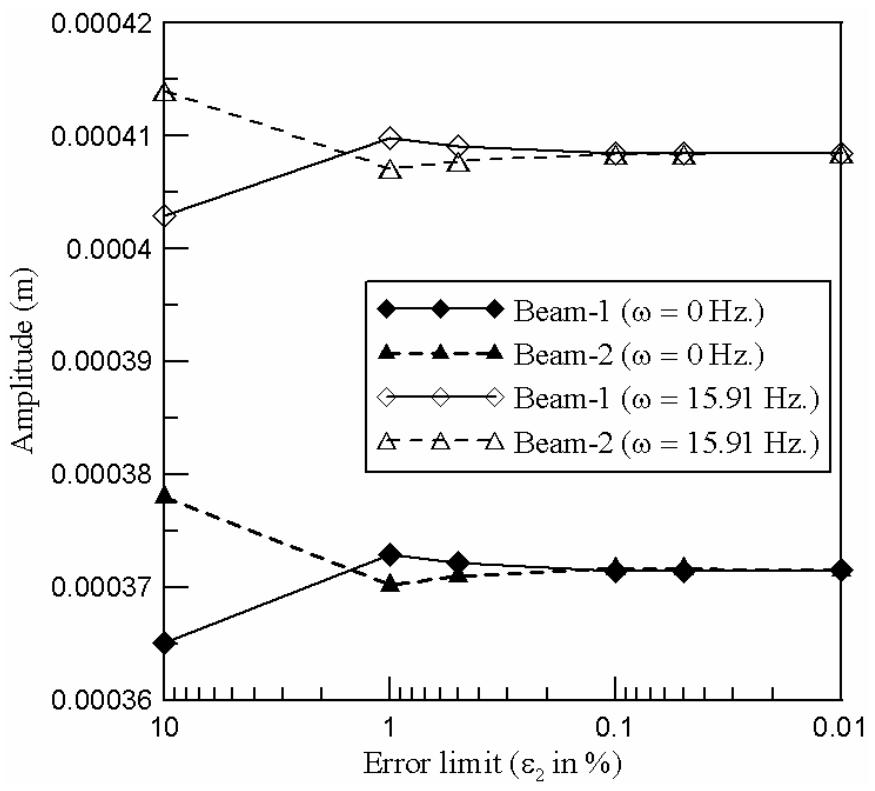

Figure 5. Convergence results for a cross-beam under uniformly distributed loading ( $\bar{p}=100 \mathrm{~N} / \mathrm{m}$ )

Validation of the present method has been carried out with the results generated in the finite element based commercial package ANSYS (ver. 11). However, ANSYS carries out the forced vibration analysis as a linear problem, whereas the present study incorporates geometric nonlinearity. Two identical beam geometries have been considered with $L_{1}=0.4 \mathrm{~m}, b_{1}=0.0247 \mathrm{~m}, t_{1}=0.003 \mathrm{~m}$ and $L_{2}=0.4 \mathrm{~m}, b_{2}=0.0247 \mathrm{~m}, t_{2}=0.003 \mathrm{~m}$ and the contact location is at $x_{r}=0.5 L_{1}$, 
$y_{r}=0.5 L_{2}$. The material properties are assumed as $E_{1}=E_{2}=210 \mathrm{GPa}, \rho_{1}=\rho_{2}=7850 \mathrm{~kg} / \mathrm{m}^{3}$. All ends of the beams have clamped boundary condition and beam-1 is under harmonic excitation of uniformly distributed type. The comparison plot is shown in Figure 6(a) for excitation amplitude $\bar{p}=10 \mathrm{~N} / \mathrm{m}$. The differences between the two sets of results are due to the differences in the basic methodologies of problem formulation, as mentioned earlier. Although ANSYS can not perform nonlinear forced vibration analysis, there is provision for inclusion of geometric nonlinearity in its static analysis. The governing equations of the system are given by, $\left[K^{i}\right]\left\{d_{i}\right\}-\omega^{2}\left[M^{i}\right]\left\{d_{i}\right\}=\left\{f_{i}\right\}, i=1$ (Beam-1), 2 (Beam-2). For $\omega=0$, the governing equation of the system becomes $\left[K^{i}\right]\left\{d_{i}\right\}=\left\{f_{i}\right\}, i=1$ (Beam-1), 2 (Beam-2). So, for a special case when $\omega=0 \mathrm{~Hz}$. the forced vibration problem reduces to a static problem which can be handled by ANSYS with incorporation of geometric nonlinearity. The solid model of crossbeam is subjected to large displacement analysis through ANSYS under static loading (i.e. $\omega=0$ ) and the results are compared in Figure 6(b). It is seen that the matching between the two sets of results is excellent.

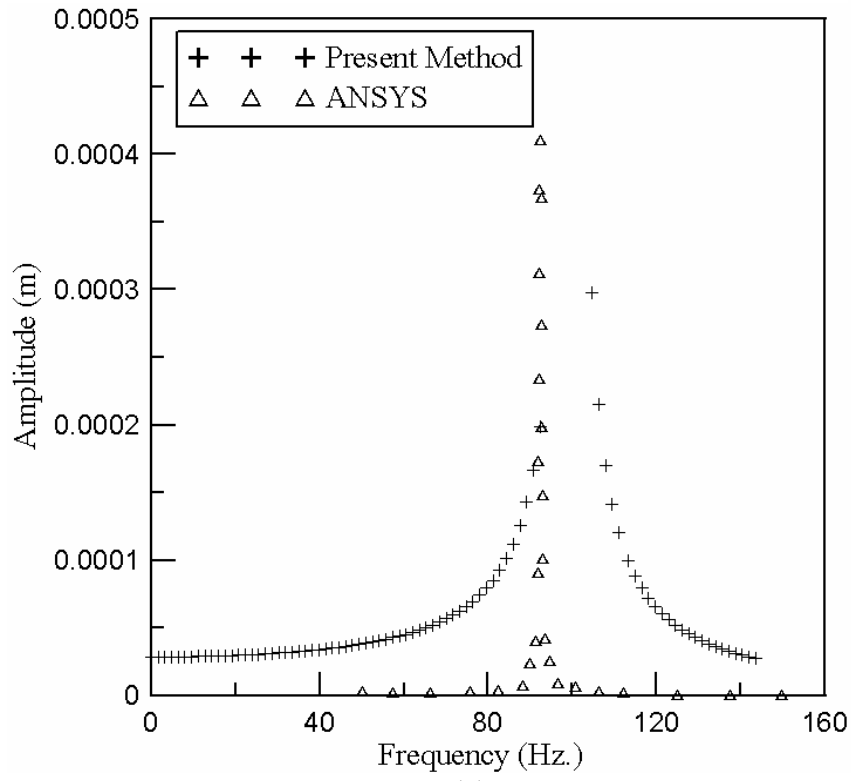

(a)

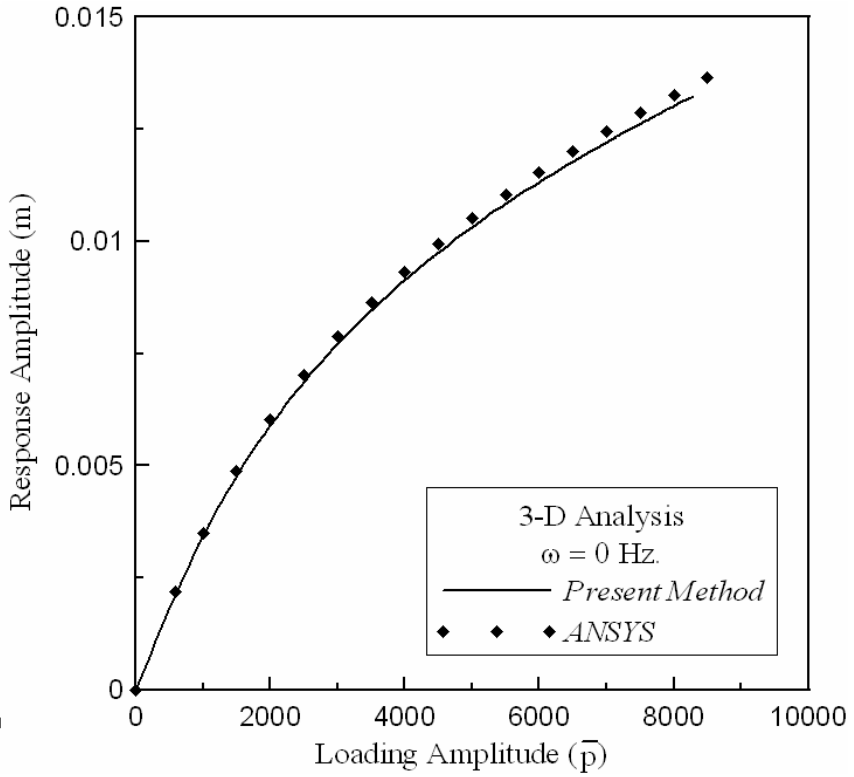

(b)

Figure 6. (a) Validation plot for a cross-beam under uniformly distributed loading ( $\bar{p}=10 \mathrm{~N} / \mathrm{m}$ ) and (b) Validation plot for a cross-beam with $\omega=0 \mathrm{~Hz}$. under uniformly distributed loading

The algorithm behind the solution methodology is already discussed in the previous section and is now depicted through Figure 7, which shows the intermediate results of each iteration step for a cross-beam under concentrated loading with amplitude $(\bar{P})$ of $100 \mathrm{~N}$. This particular set of results are generated for an excitation frequency of $0 \mathrm{~Hz}$., which signifies an equivalent static deflection scenario. The dimensions of the system are $L_{1}=1 \mathrm{~m}, b_{1}=0.02 \mathrm{~m}, t_{1}=0.01 \mathrm{~m}$ and $L_{2}=1 \mathrm{~m}, b_{2}=0.02 \mathrm{~m}, t_{2}=0.01 \mathrm{~m}$ and contact location is $x_{r}=0.5 L_{1}, y_{r}=0.5 L_{2}$. The material properties are assumed as $E_{1}=E_{2}=210 \mathrm{GPa}, \rho_{1}=\rho_{2}=7850 \mathrm{~kg} / \mathrm{m}^{3}$. Figure 7(a) plots the fraction $(q)$ of excitation amplitude against iteration number. This fraction $(q)$ is the controlling parameter of the iterative procedure and depending on the value of $q$ the vibration amplitudes of the two beams are calculated. These values are shown and compared in Figure 7(b). Finally, Figure 7(c) furnishes the error in each iteration signifying the difference between the amplitudes of the two beams. At the start of the iteration (Iteration no. 1) calculations are based on a guess value of $q$, which in this case is far from the exact value and hence the error is maximum. At this stage it is seen that the amplitude value of beam- 1 is greater than beam- 2 and according to the algorithm $q_{\min }$ is upgraded to this $q$ value. After the modification of the limit, the new value $q$ is determined using the following expression, $q=q_{\min }+q_{1}\left(q_{\max }-q_{\min }\right)$. The second step of the iteration starts with this newly determined value of $q$ and the same calculations are carried out. The process proceeds the same way until the error value comes down below the acceptable error limit $\left(\varepsilon_{2}\right)$. For this particular frequency 17 iterations are required to achieve the desired convergence. 


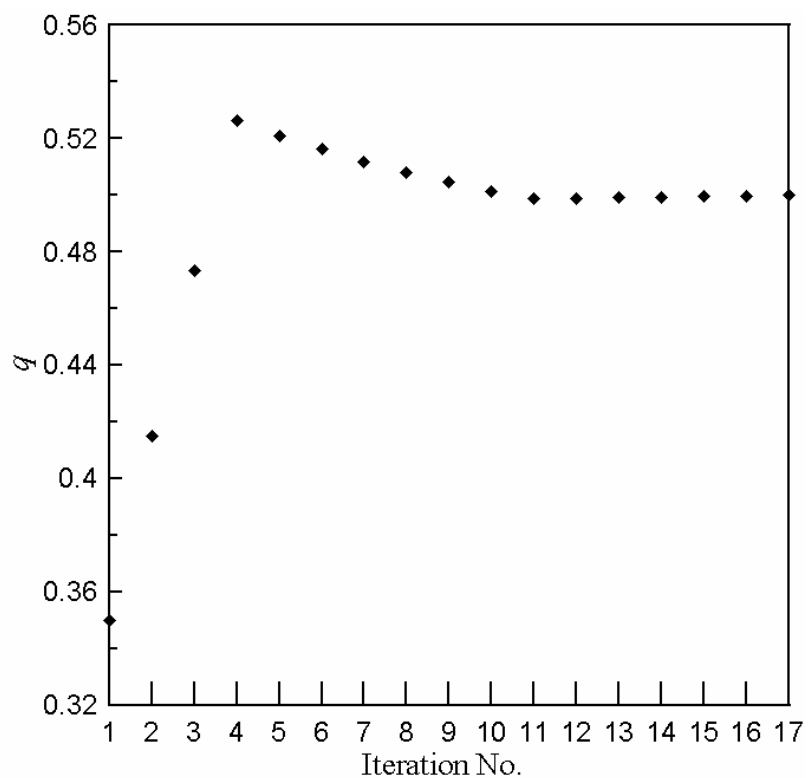

(a)

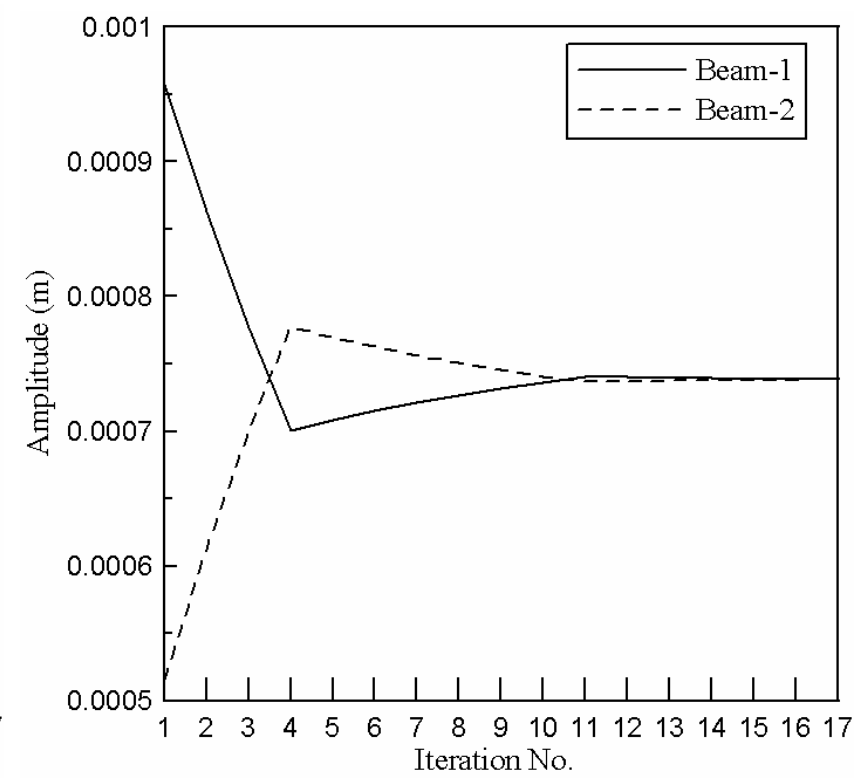

(b)

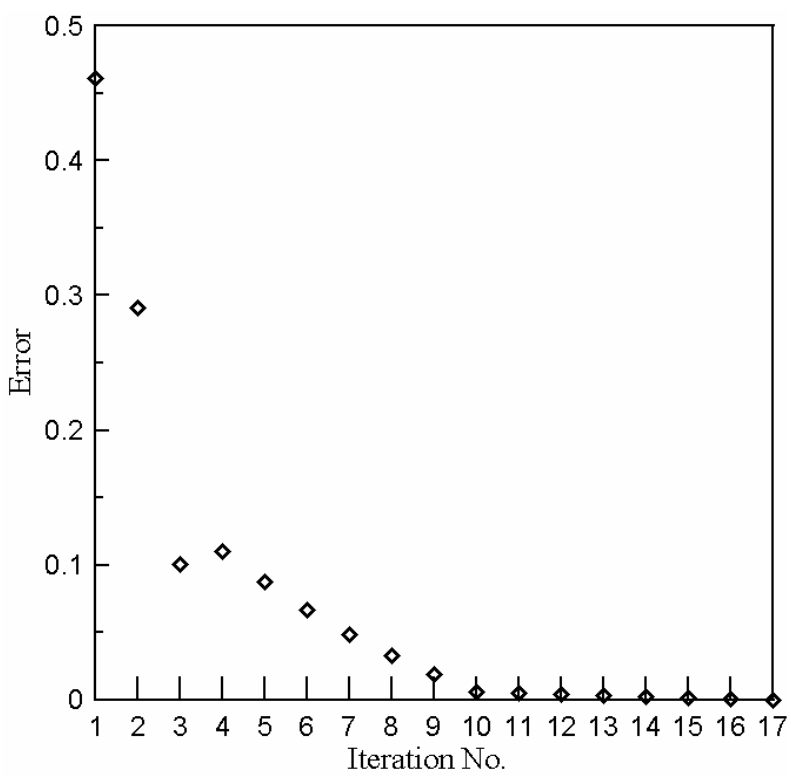

(c)

Figure 7. Plots of (a) fraction ( $q$ ) of total excitation amplitude, (b) amplitudes of beam vibration and (c) error (= abs $\left.\left\{\left(\delta_{b 1}-\delta_{b 2}\right) / \delta_{b 1}\right\}\right)$ against iteration number for a cross-beam under concentrated harmonic excitation $(\bar{P}=100 N)$ at zero frequency

The frequency response curves in dimensional amplitude-frequency plane are presented for contact location $x_{r}=0.5 L_{1}$, $y_{r}=0.5 L_{2}$ in Figure 8(a)-(d) for concentrated load, uniformly distributed load, triangular load and hat type load respectively. In each of these plots, response curves are presented for three different values (low, medium and high) of excitation amplitudes. Results are generated using the following geometric and material parameter values: Dimensions: $L_{1}=1 \mathrm{~m}, b_{1}=0.02 \mathrm{~m}, t_{1}=0.01 \mathrm{~m}$ and $L_{2}=1 \mathrm{~m}, b_{2}=0.02 \mathrm{~m}, t_{2}=0.01 \mathrm{~m}$, Material property: $E_{1}=E_{2}=210 \mathrm{GPa}, \rho_{1}=\rho_{2}=7850 \mathrm{~kg} / \mathrm{m}^{3}$. It must be noted that the response curves are presented only for the first mode of vibration and correspond to primary resonance condition where the external excitation frequency becomes equal to the lowest fundamental frequency of the system. 


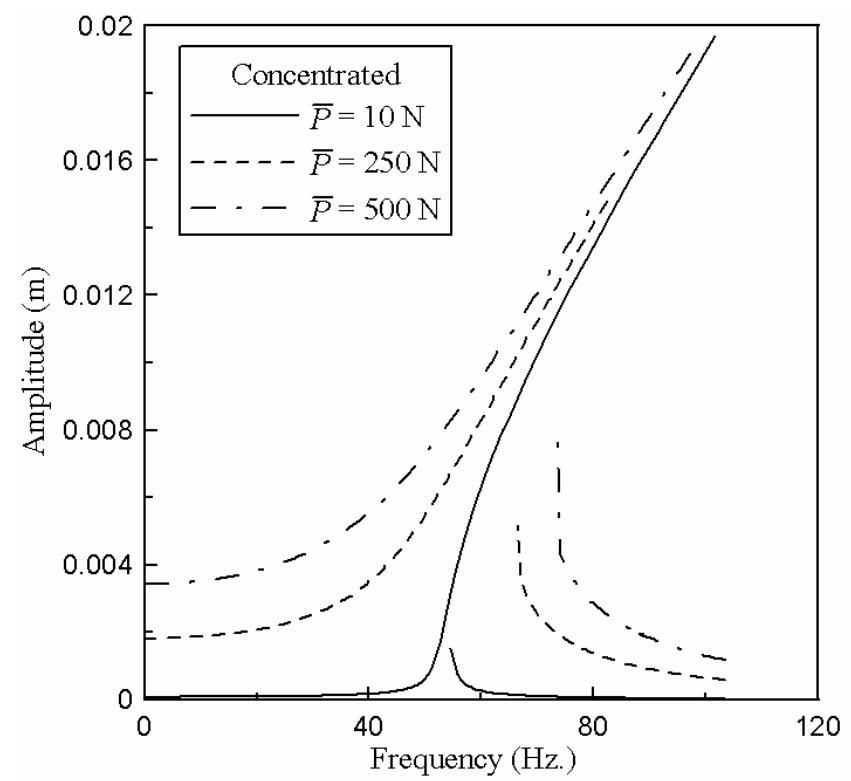

(a)

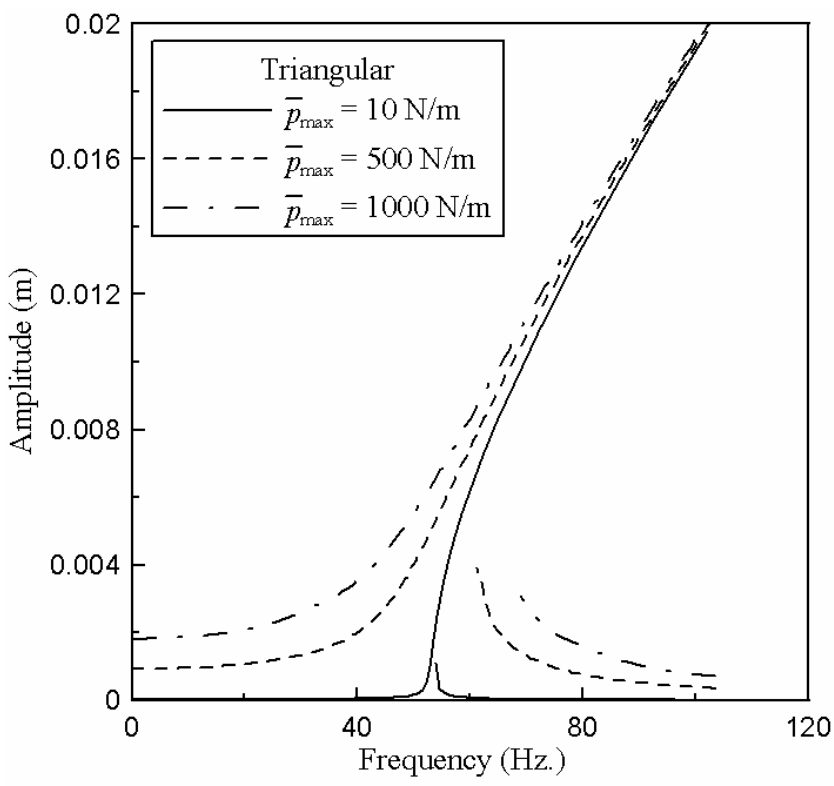

(c)

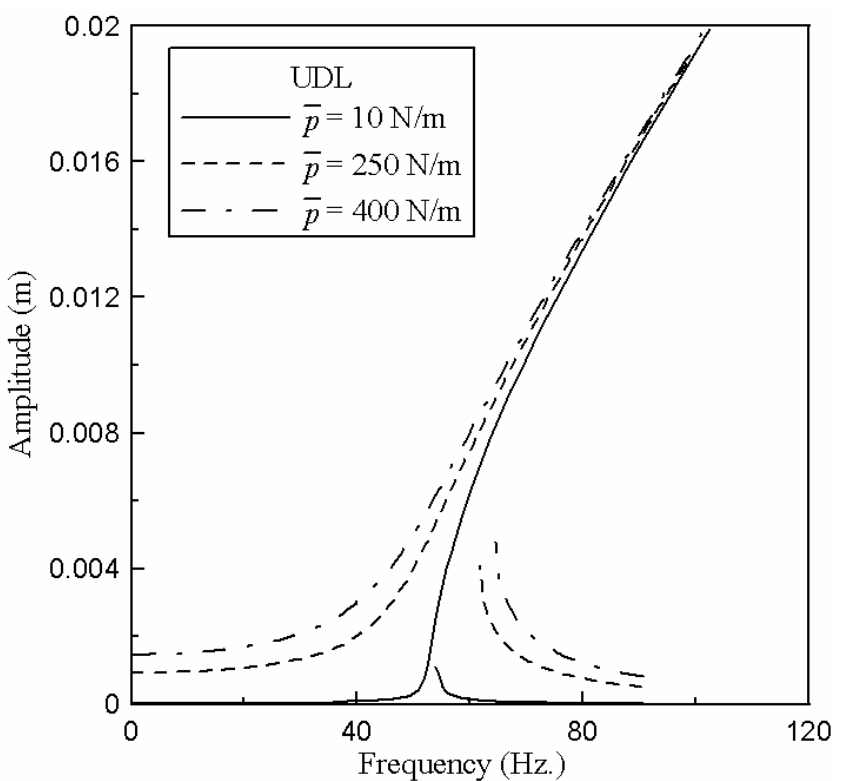

(b)

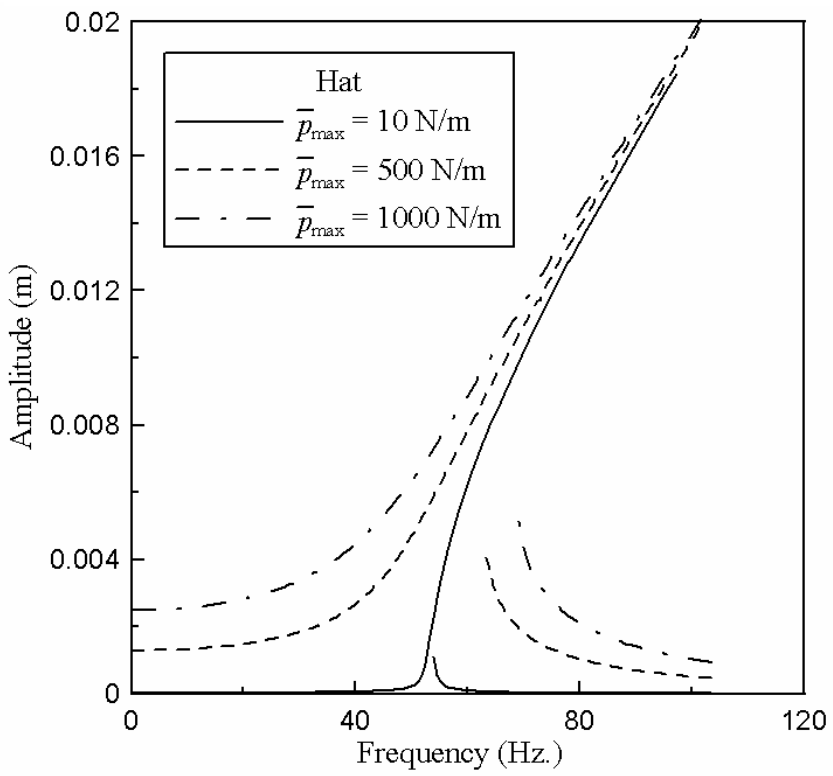

(d)

Figure 8. Frequency response curves of cross-beam with contact location $x_{r}=0.5 L_{1}, y_{r}=0.5 L_{2}$ under different types of loading: (a) Concentrated, (b) UDL, (c) Triangular and (d) Hat.

From the set of figures it is clear that the system exhibits hardening type nonlinearity, which means the free vibration frequency increases with increase in vibration amplitude. Although a separate free vibration analysis of the system is not carried out in course of the present analysis, the nature of backbone curve can be surmised from the forced vibration frequency response curves, as these curves envelope the backbone curve. As the amplitude of excitation is reduced the nonlinear response of the system tends to attain the shape of backbone curve. For all the different cases of loading each response curve shows two distinct regions. At low values of the excitation frequency the system response is unique and the vibration amplitude increases monotonically with increase in excitation frequency. But after a certain excitation frequency two response amplitudes are obtained and there exists two branches of response amplitude over a certain frequency range. The amplitude of the initial branch continues to increase with excitation frequency from the single valued region, whereas the later branch shows a reverse trend as the vibration amplitude decreases with increase in frequency. The static response of the system can also be determined from the location where the response curve intersects the vertical axis, which signifies zero excitation frequency. This point denotes the static deflection of the cross-beam under loading corresponding to the excitation amplitude. It is known that nonlinear systems may exhibit super-harmonic and sub- 
harmonic responses, but for the given set of system parameters and loading conditions these nonlinear phenomena are not manifested.

It is noted in course of solution that if direct substitution method with successive relaxation scheme is used then for ascending sweep of excitation frequency only a part of the initial higher branch of the response amplitude is captured. However, this technique is able to capture the next lower branch of the response completely. On the other hand Broyden's method captures the higher branch completely when excitation frequency is swept up and the lower branch is obtained by sweeping the frequency from higher to lower values.

Theoretically the response curve for a harmonic excitation is made up of three regions, of which two are stable and experimentally obtainable. Actually, in the multi-valued interval of the response there are three states of solution for a given excitation frequency. But the middle region upto the saddle point corresponds to an unstable response and cannot be captured experimentally. The present analysis captures the two stable regions but could not capture the unstable region. In these two stable zones the system response increases with increase in excitation amplitude. This fact also differentiates the two stable regions from the unstable one as in that zone the response reduces with increase in excitation amplitude.

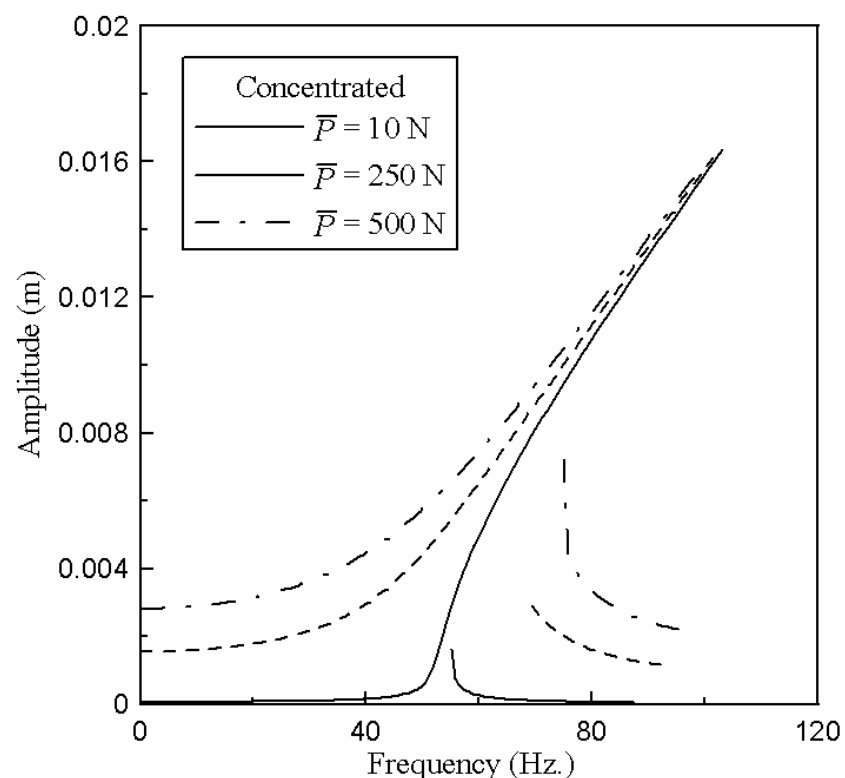

(a)

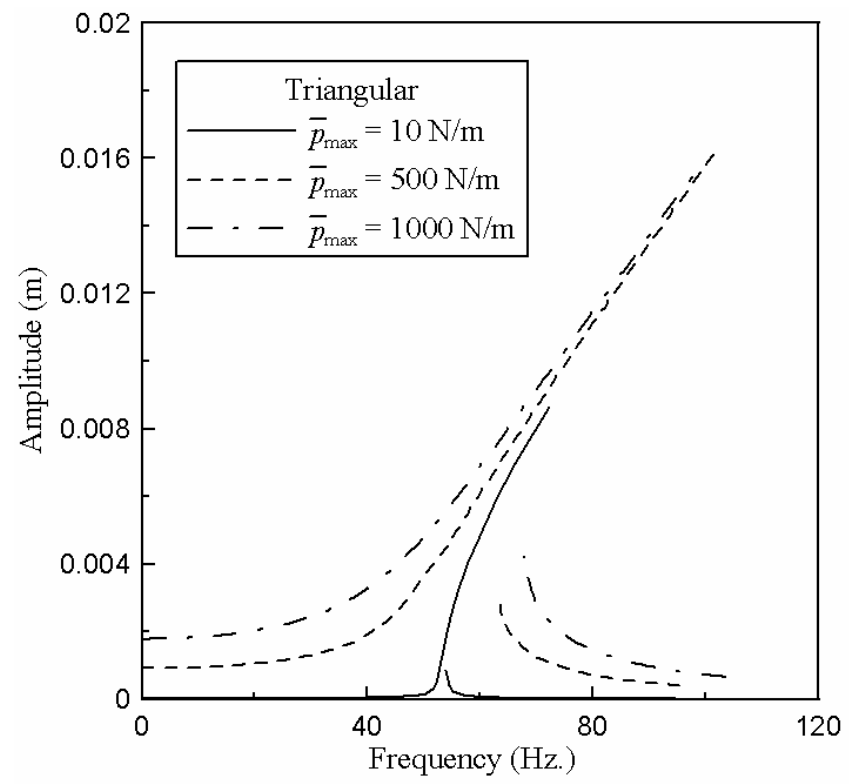

(c)

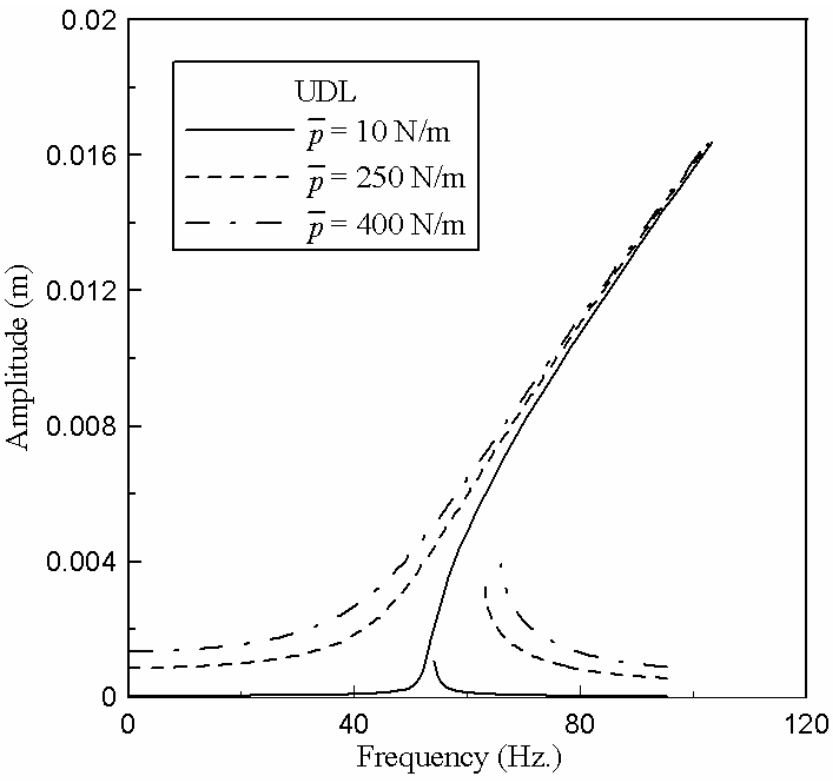

(b)

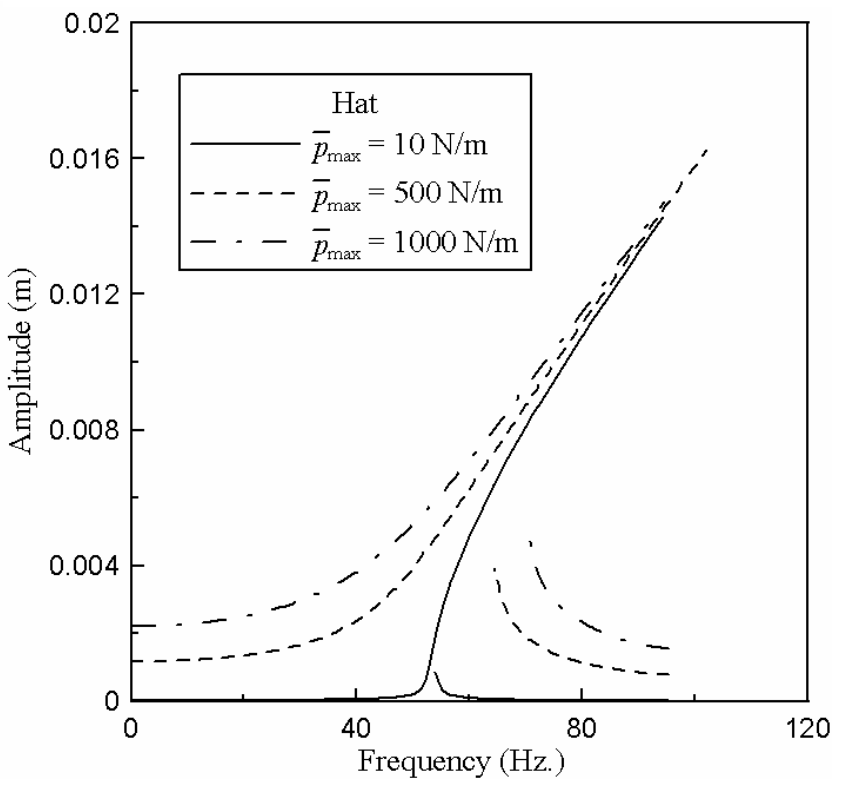

(d)

Figure 9. Frequency response curves of cross-beam with contact location $x_{r}=0.333 L_{1}, y_{r}=0.5 L_{2}$ under different types of loading: (a) Concentrated, (b) UDL, (c) Triangular and (d) Hat. 


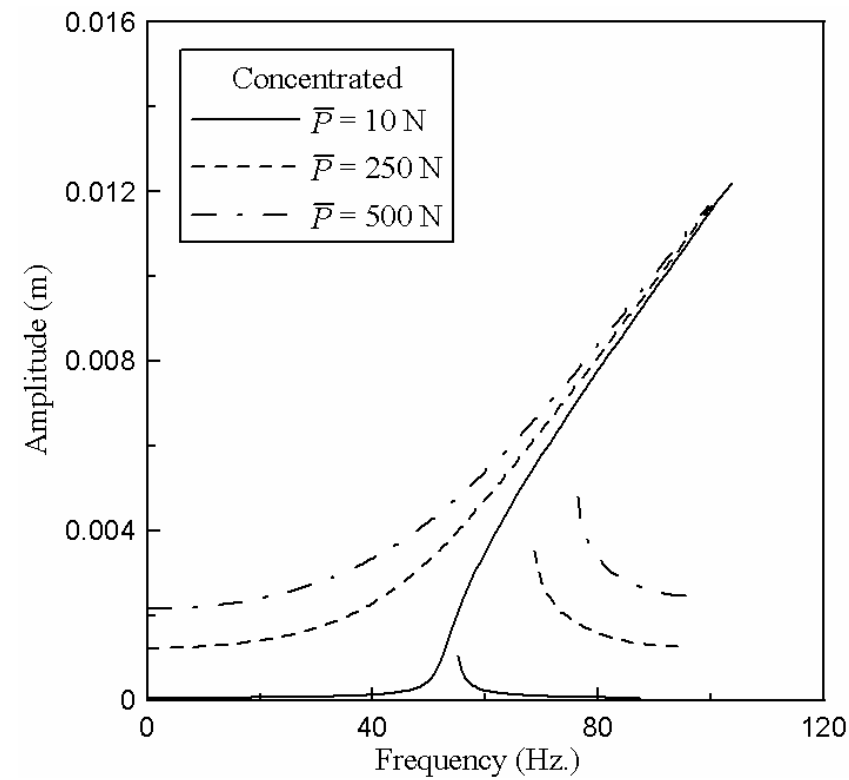

(a)

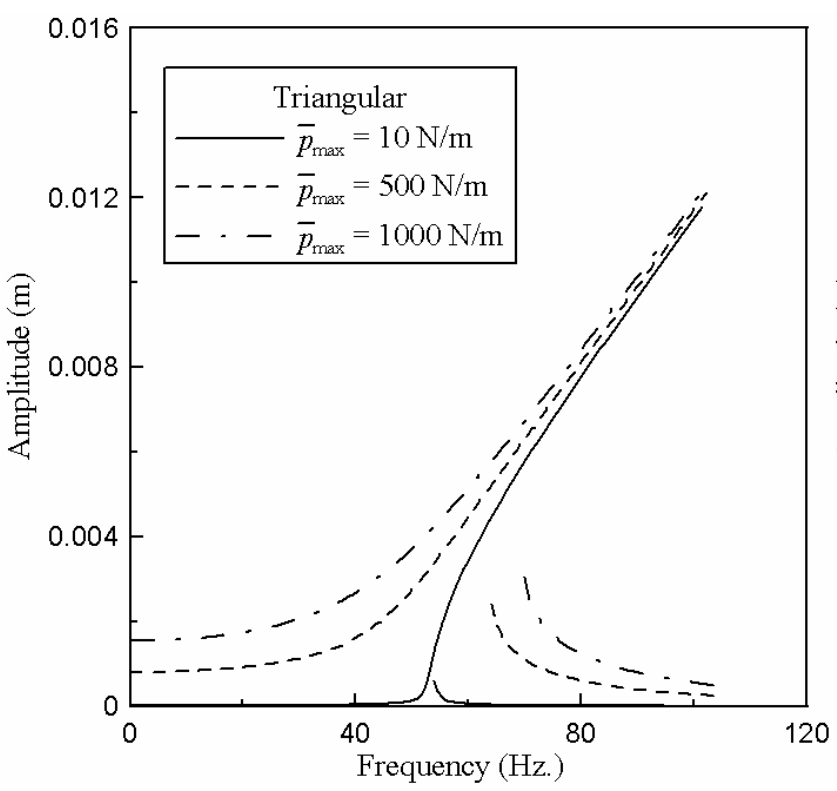

(c)

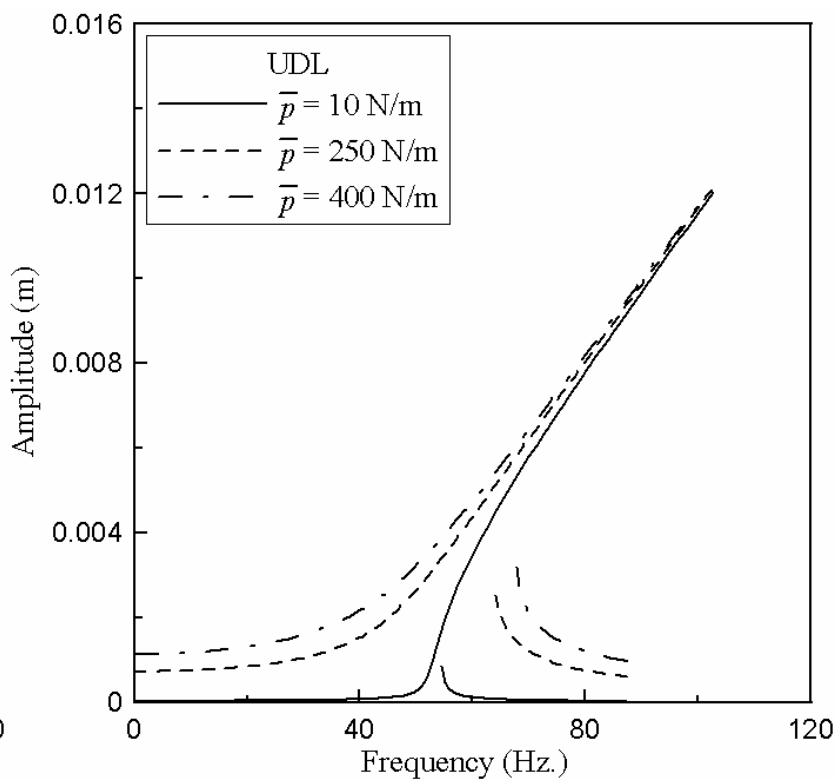

(b)

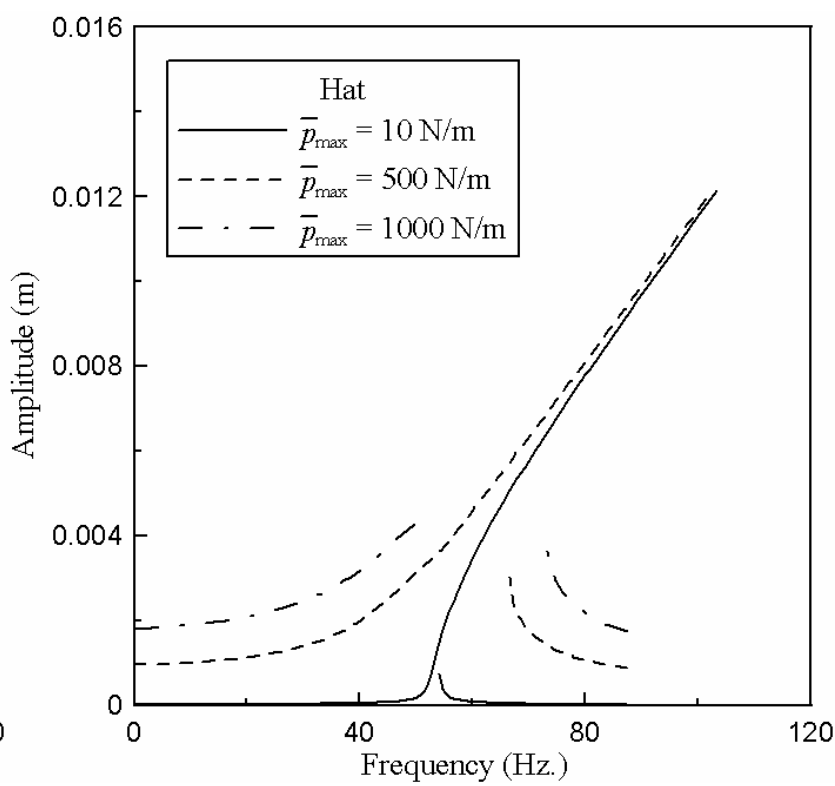

(d)

Figure 10. Frequency response curves of cross-beam with contact location $x_{r}=0.25 L_{1}, y_{r}=0.5 L_{2}$ under different types of loading: (a) Concentrated, (b) UDL, (c) Triangular and (d) Hat.

Frequency-amplitude response curves of the cross-beam system under different harmonic loading patterns for two different contact locations, $x_{r}=0.333 L_{1}, y_{r}=0.5 L_{2}$ and $x_{r}=0.25 L_{1}, y_{r}=0.5 L_{2}$ are presented in Figure 9 and 10 respectively. These figures also depict similar characteristics as those discussed in relation to Figure 8. It should be noted that the amplitude of vibration presented as the ordinate of the response curves correspond to the contact point of the two beams. Comparison of figures 8, 9 and 10 reveals that the response amplitude of the contact point decreases as the position of the lower beam shifts away from the center. This is more clearly demonstrated in Figure 11, which shows the response plots for variation in contact location for various types of loading. It should also be mentioned that the contact point of the beams may not necessarily be the point of maximum defection amplitude. In particular, asymmetry in loading (e.g., triangular) and geometry (e.g., $x_{r}=0.25 L_{1}$ ) may cause the point of maximum deflection to shift away from the center of the beam. For example, when a concentrated type excitation is applied over the contact point of two beams, irrespective of the geometry the maximum deflection of the system occurs at that point. But for symmetrically distributed excitation (UDL and Hat load) the geometry of the cross-beam becomes important in 
determining the point of maximum deflection. For concentrated excitation applied at locations other than the contact point, the maximum deflection is likely to occur away from the contact point of the beams.

From Figure 11 it is observed that for concentrated loading at low excitation frequency response corresponding to $x_{r}=0.5 L_{1}$ is highest and that due to $x_{r}=0.25 L_{1}$ is lowest. It is also seen that the response curves exhibit a small difference between themselves. For UDL also response is higher due to $x_{r}=0.5 L_{1}$, but the gap between the three curves has diminished. Finally, for triangular and hat type loading the gap between the response curves is so small that they cannot be distinguished visually. At higher excitation frequencies the higher branches of the responses are clearly distinguishable and throughout all type of loadings the highest response occurs for the symmetric geometry i.e. $x_{r}=0.5 L_{1}, y_{r}=0.5 L_{2}$. The lower branches of the amplitude response are very close together for all the cases.

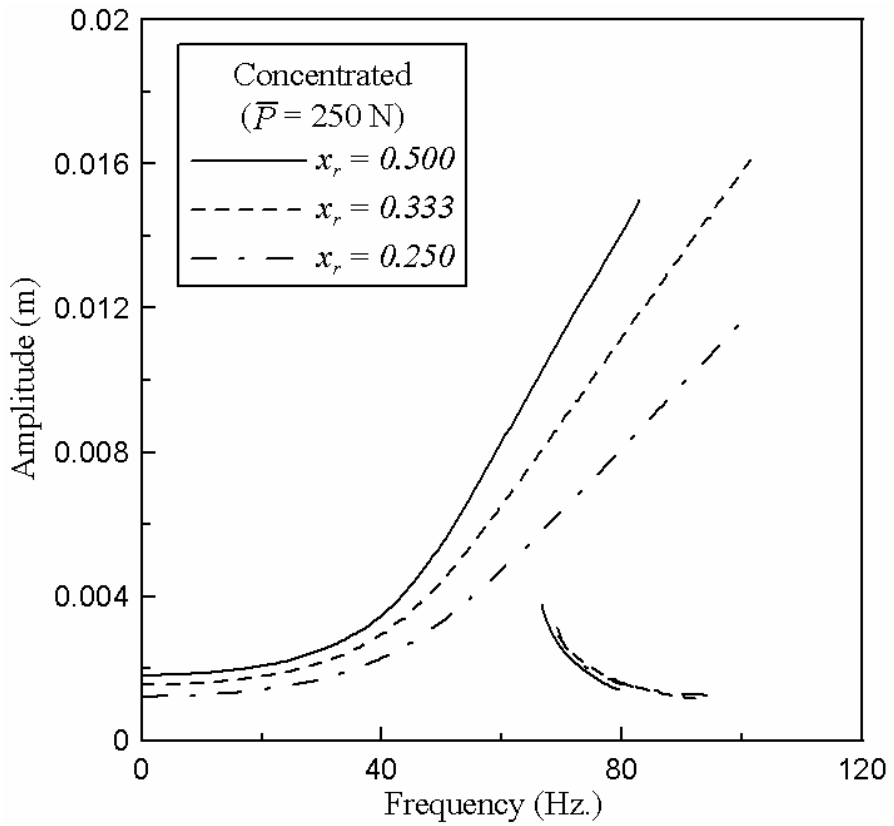

(a)

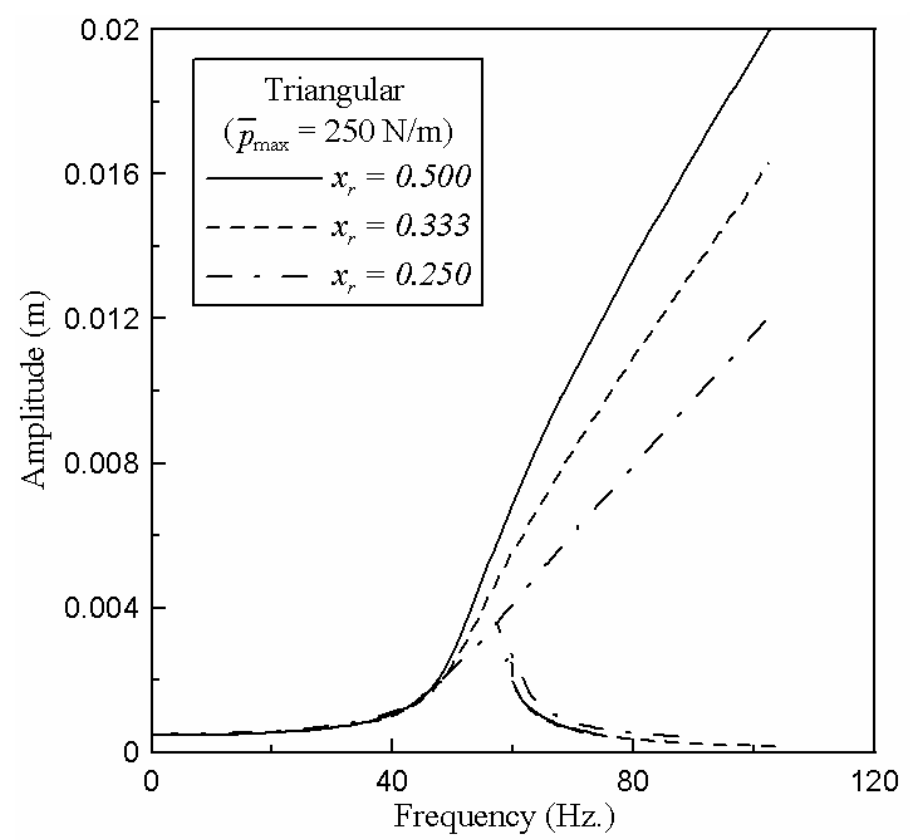

(c)

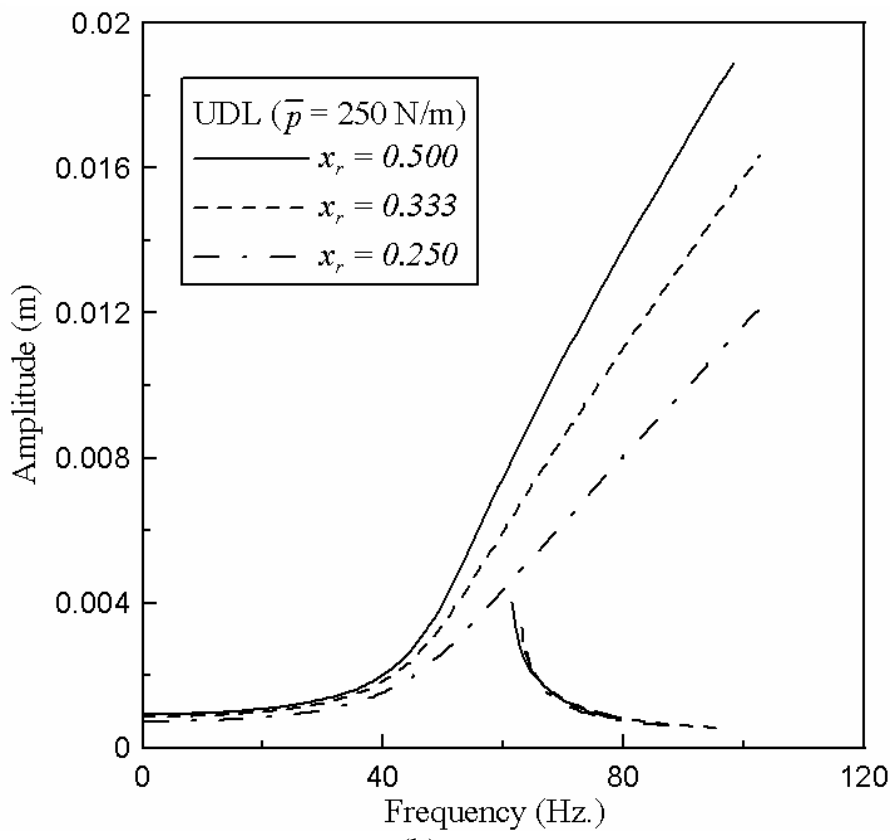

(b)

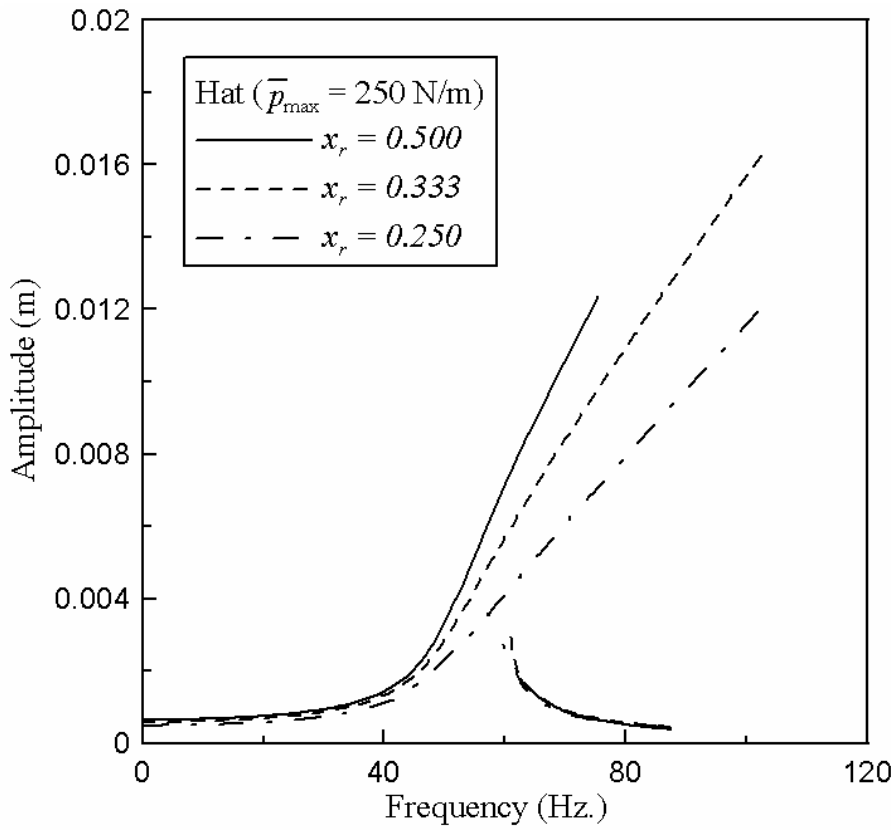

(d)

Figure 11. Frequency response curves of cross-beam for variation of contact location under different types of loading: (a) Concentrated, (b) UDL, (c) Triangular and (d) Hat. 
A study on the effect of variation of thickness of beam-2 on the system response is also carried out. Figure 12 presents the effect of variation of beam-2 thickness for concentrated loading and contact location of $x_{r}=0.5 L_{1}, y_{r}=0.5 L_{2}$ at three different load levels. From the figure it is clear that whenever beam- 2 thickness is not equal to beam- 1 thickness, there exists a discontinuity on the increasing branch of the response. It is noted that the discontinuity occurs around the natural frequency of the thinner beam. Physically it signifies that near its natural frequency the response of the thinner beam increases rapidly, but the thicker beam not yet near its natural frequency can not replicate the behaviour of the other beam.

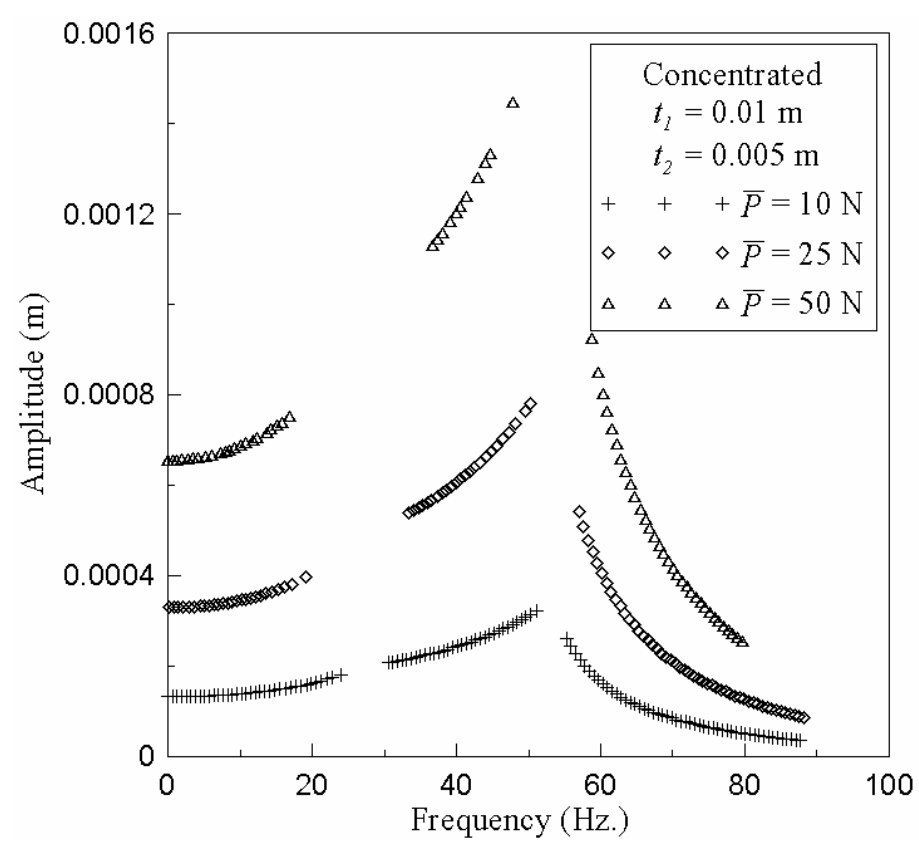

(a)

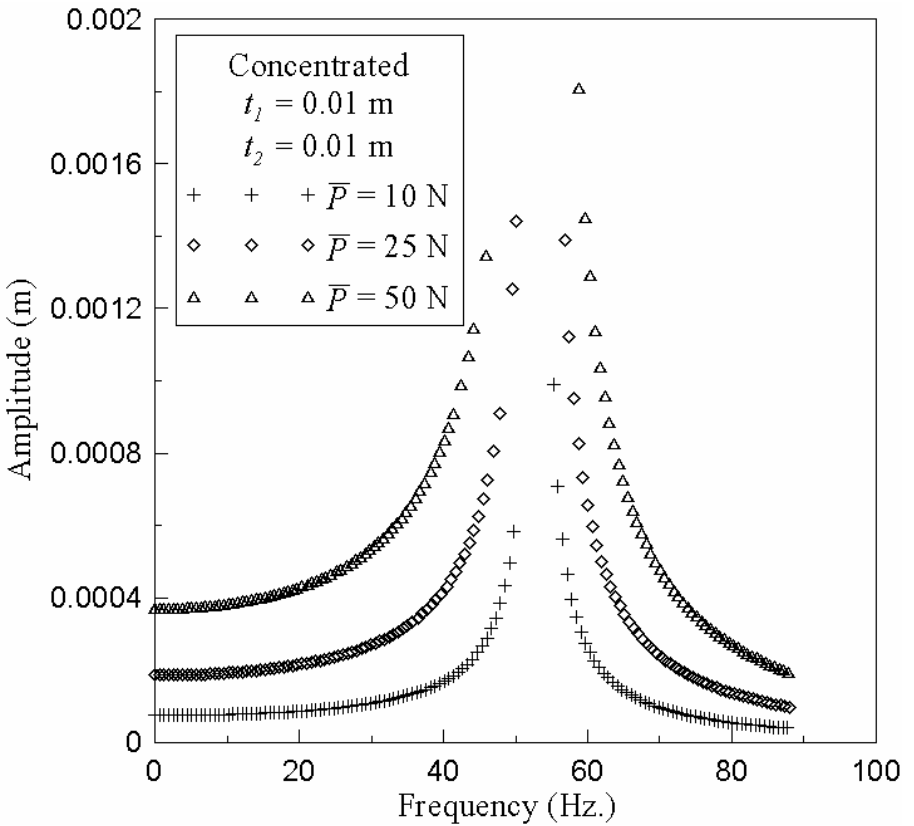

(b)

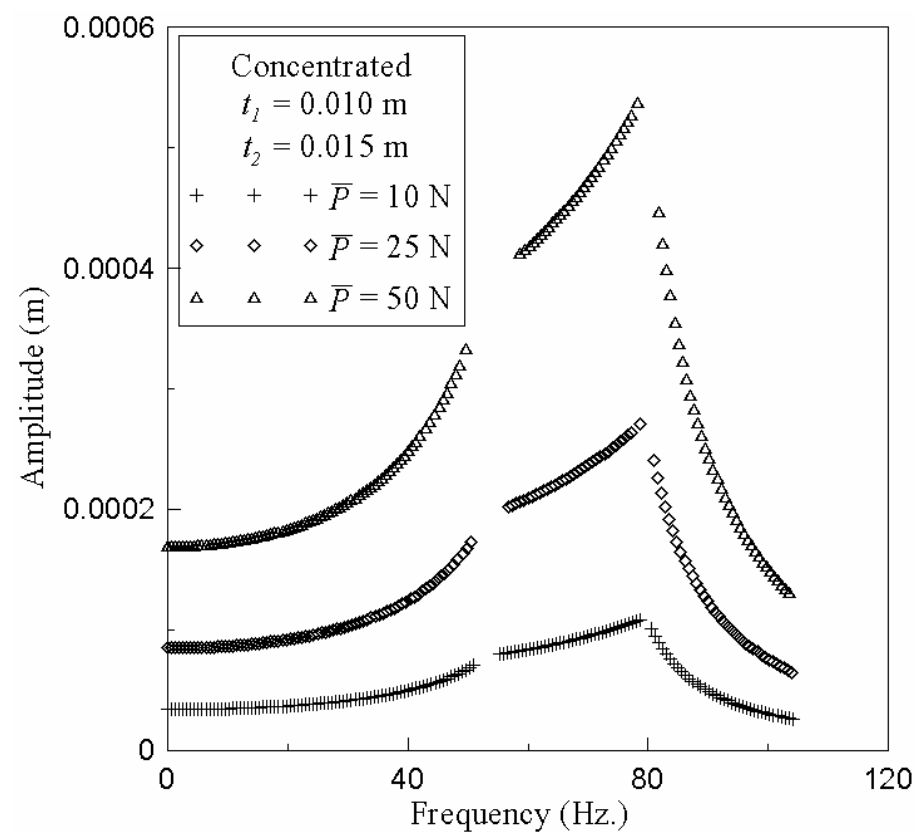

(c)

Figure 12. Frequency response curves of cross-beam with contact location $x_{r}=0.5 L_{1}, y_{r}=0.5 L_{2}$ under concentrated loading for variation of beam-2 thickness: (a) $t_{2}=0.005 \mathrm{~m}$ (b) $t_{2}=0.010 \mathrm{~m}$ and (c) $t_{2}=0.015 \mathrm{~m}$. 
It is also important to note that with increase in the frequency of excitation, the magnitude of the dynamic system stiffness ( $\left[K^{i}-\omega^{2} M^{i}\right], i=1,2$ ) may become negative when the excitation frequency becomes greater than the natural frequency of the beam. Under such situations the phase of the load vector is reversed and mode of vibration is also reversed. Hence, for a crossbeam with two beams of different thickness at a certain excitation frequency (greater than natural frequency of thinner beam but lower than the natural frequency of the thicker beam) the two beams may vibrate out of phase and thus give rise to the discontinuous response. Beyond the natural frequency of the thicker beam the two beams again vibrate in phase as the mode of vibration of the second beam is also reversed. Interestingly, beyond this frequency the response curves presented in Figure 12 do not show any discontinuity.

\section{Conclusions}

In the present paper large displacement forced vibration analysis of a cross-beam system is presented using an energy method and variational formulation. The forced vibration analysis is carried out in an indirect way, in which the dynamic system is assumed to satisfy the force equilibrium condition at maximum load value, thus reducing the problem to an equivalent static case. The two beams of the system are solved individually either by a direct substitution method employing successive relaxation technique or a multi-dimensional secant method, known as Broyden's method. An iterative scheme, based on the reaction force between the two beams of the system and equality of vibration amplitude at the contact of two beams, is developed to obtain the response of the system under external harmonic excitation. The present method is validated through results generated in ANSYS 11.0. Results are provided to establish the working methodology of the iterative procedure. The system response is studied for variation of loading type and contact location of the beams, achieved by changing the position of the lower beam. The results are presented in terms of plots of vibration amplitude of the contact point between the two beams versus frequency of forcing excitation in dimensional plane. The system responses indicate a hardening type nonlinearity and two stable zones of the response curve is obtained. It is seen that an increase in excitation amplitude corresponds to increase in response amplitude. Results also indicate that the vibration amplitude of the contact point decreases as the contact shifts away from the center. The system response is studied for variation in thickness of the lower beam and it is observed that the response curves show a discontinuity around the natural frequency of the thinner beam. An effort is made to explain the discontinuous nature of the responses.

\section{Nomenclature}

$\begin{array}{ll}A_{1}, A_{2} & \text { Cross-sectional area of beam- } 1 \text { and beam- } 2 \\ b_{1}, b_{2} & \text { Width of beam-1 and beam-2 } \\ \left\{d_{1}\right\},\left\{d_{2}\right\} & \text { Vector of unknown coefficients } \\ E_{1}, E_{2} & \text { Young's modulus of the beam material } \\ I_{1}, I_{2} & \text { Second Moment of area } \\ \left\{f_{1}\right\},\left\{f_{2}\right\} & \text { Load vectors } \\ {\left[K^{1}\right],\left[K^{2}\right]} & \text { Stiffness matrix } \\ {\left[K_{b}^{1}\right],\left[K_{b}^{2}\right]} & \text { Stiffness matrix due to bending } \\ {\left[K_{m}^{1}\right],\left[K_{m}^{2}\right]} & \text { Stiffness matrix due to stretching } \\ L & \text { Lagrangian } \\ L_{1}, L_{2} & \text { Length of the beams } \\ {\left[M^{1}\right],\left[M^{2}\right]} & \text { Mass Matrix } \\ n w & \text { Number of functions for } w_{1}, w_{2} \\ n u & \text { Number of functions for } u_{1}, u_{2} \\ P & \text { Harmonically varying transverse concentrated loading on beam-1 } \\ \bar{P} & \text { Amplitude of harmonic excitation for concentrated loading } \\ p & \text { Harmonically varying transverse pressure type loading on beam-1 } \\ \bar{p} & \text { Amplitude of harmonic excitation for pressure type loading } \\ q & \text { Ratio of reaction force between the two beams and total load on beam-1 } \\ q_{1} & \text { Load distribution factor } \\ R & \text { Reaction force generated between the two beams } \\ \bar{R} & \text { Amplitude of the reaction } \\ T_{1}, T_{2} & \text { Kinetic energy of beam- } 1 \text { and beam-2 }\end{array}$




$\begin{array}{ll}t_{1}, t_{2} & \text { Thickness of the beams } \\ u_{1}, u_{2} & \text { Dynamic displacement along axial direction of the beams } \\ U_{1}, U_{2} & \text { Strain energy of beam-1 and beam-2 } \\ U_{b 1}, U_{b 2} & \text { Strain energy due to bending of beam- } 1 \text { and beam-2 } \\ U_{m 1}, U_{m 2} & \text { Strain energy due to stretching of beam- } 1 \text { and beam-2 } \\ V_{1}, V_{2} & \text { Work potential for external load of beam-1 and beam-2 } \\ w_{1}, w_{2} & \text { Dynamic transverse displacement } \\ x_{p} & \text { Point of application of concentrated loading on beam-1 } \\ x_{r} & \text { Location on beam-1 where the reaction force is acting } \\ y_{r} & \text { Location on beam-2 where the reaction force is acting } \\ \alpha_{1 i}, \alpha_{2 i} & \text { Set of functions defining approximate displacement field } u_{1}, u_{2} \\ \delta_{b 1}, \delta_{b 2} & \text { Transverse displacement amplitude of beam- } 1 \text { and beam- } 2 \text { at the contact point } \\ \delta & \text { Variational operator } \\ \varepsilon_{X}^{b} & \text { Axial strain of beam due to bending } \\ \varepsilon_{x}^{s} & \text { Axial strain of beam due to stretching } \\ \phi_{1 i}, \phi_{2 i} & \text { Set of functions defining approximate displacement field } w_{1}, w_{2} \\ \eta_{r} & \text { Normalized location on beam-2 where the reaction force is acting } \\ \pi & \text { Total energy of the system } \\ \rho_{1}, \rho_{2} & \text { Density of the beam materials } \\ \tau & \text { Time coordinate } \\ \omega & \text { Frequency of excitation } \\ \omega_{1} & \text { First linear natural frequency of vibration } \\ \xi_{p} & \text { Normalized axial coordinate of point of application of concentrated loading on beam-1 } \\ \xi_{r} & \text { Normalized location on beam- } 1 \text { where the reaction force is acting }\end{array}$

\section{References}

Abu-Hilal M. 2003. Forced vibration of Euler-Bernoulli beams by means of dynamic Green functions. Journal of Sound and Vibration, vol. 267, pp. 191-207.

Arboleda-Monsalve L.G., Zapata-Medina D.G. and Aristizabal-Ochoa J.D. 2007. Stability and natural frequencies of a weakened Timoshenko beam-column with generalized end conditions under constant axial load. Journal of Sound and Vibration, vol. 307, No. 1-2, pp. 89-112.

Aristizabal-Ochoa J.D. 2004. Timoshenko Beam-Column with Generalized End Conditions and Nonclassical Modes of Vibration of Shear Beams. ASCE Journal of Engineering Mechanics, vol. 130, No. 10, pp. 1151-1159.

Azrar L. Benamar R. and White R.G. 1999. A semi-analytical approach to the nonlinear dynamic response problem of S-S and C$\mathrm{C}$ beams at large vibration amplitudes. Part I: general theory and application to the single mode approach to free and forced vibration analysis. Journal of Sound and Vibration, vol. 224, No. 2, pp. 183-207.

Azrar L. Benamar R. and White R.G. 2002. A semi-analytical approach to the non-linear dynamic response problem of beams at large vibration amplitudes, Part II: multimode approach to the steady state forced periodic response. Journal of Sound and Vibration, vol. 255, No. 1, pp. 1-41.

Bennett J.A. and Eisley J.G. 1970. A multiple degree-of-freedom approach to nonlinear beam vibrations. AIAA Journal, vol. 8, pp. 734-739.

Chen S. Ma A.J. and Han W. 1994. Design sensitivity analysis of nonlinear mode spectrum for large amplitude forced vibrations of beams. Computers \& Structures, vol. 53, No. 2, pp. 363-366.

Das D. Sahoo P. and Saha K.N. 2007. Large amplitude forced vibration analysis of thin beams for different boundary conditions and loading pattern. Proceedings of 52nd Congress of The Indian Society of Theoretical and Applied Mechanics, 2007, Bangalore, pp. 43-50.

Doong J.L. and Chen C.S. 1988. Large amplitude vibration of a beam based on a higher-order deformation theory. Applied Acoustics, vol. 25, No. 4, pp. 281-293.

Evensen D.A. 1968. Nonlinear vibrations of beam with various boundary conditions. AIAA Journal, vol. 6, pp. 370-372. 
Ewing M.S. and Mirsafian S. 1996. Forced vibration of two beams joined with a non-linear rotational joint: clamped and simply supported end conditions. Journal of Sound and Vibration, vol. 193, No. 2, pp. 483-496.

Fan Z.J. Lee J.H. Kang K.H. and Kim K.J. 1998. The forced vibration of a beam with viscoelastic boundary supports. Journal of Sound and Vibration, vol. 210, No. 5, pp. 673-682.

Fung R.F. and Chen C.C. 1997. Free and forced vibration of a cantilever beam contacting with a rigid cylindrical foundation. Journal of Sound and Vibration, vol. 202, No. 2, pp. 161-185.

Kadiri M.E. Benamer R. and White R.G. 2002. Improvement of the semi-analytical method, for determining the geometrically non-linear response of thin straight structures. Part I: application to clamped-clamped and simply supported-clamped beams. Journal of Sound and Vibration, vol. 249, No. 2, pp. 263-305.

Leissa A.W. 1989. Closed form exact solutions for the steady state vibrations of continuous systems subjected to distributed exciting forces. Journal of Sound and Vibration, vol. 134, pp. 435-453.

Ma A.J. Chen S. and Liu Z. 1995a. Statistical properties of nonlinear mode spectrum for large amplitude forced vibration systems. Computers \& Structures, vol. 56, No. 6, pp. 897-902.

Ma A.J. Chen S. and Song D.T. 1995b. A new method of nonlinear response analysis for large deflection forced vibrations of beams. Finite Elements in Analysis and Design, vol. 20, pp. 39-46.

Maccari A. 1999. The Asymptotic Perturbation Method for Nonlinear Continuous Systems. Nonlinear Dynamics. vol. 19, pp. 118.

Marur S.R. 2001. Advances in nonlinear vibration analysis of structures. Part-I. Beams. Sadhana, vol. 26, No. 3, pp. 243-249.

Mei C. and Decha-Umphai K. 1985. A finite element method for non-linear forced vibrations of beams. Journal of Sound and Vibration, vol. 102, No. 3, pp. 369-380.

Meirovitch L. 1970. Methods of Analytical Dynamics. McGraw Hill Inc.

Oniszczuk Z. 2003. Forced transverse vibrations of an elastically connected complex simply supported double-beam system. Journal of Sound and Vibration, vol. 264, pp. 273-286.

Ozkaya E. 2002. Non-linear transverse vibrations of a simply supported beam carrying concentrated masses. Journal of Sound and Vibration, vol. 257, No. 3, pp. 413-424.

Ribeiro P. 2004. Non-linear forced vibrations of thin/thick beams and plates by the finite element and shooting methods. Computers \& Structures, vol. 82, pp. 1413-1423.

Sapountzakis E.J and Dourakopoulos J.A. 2009. Nonlinear dynamic analysis of Timoshenko beams by BEM. Part I: Theory and numerical implementation. Nonlinear Dynamics. vol. 58, No. 1, pp. 295-306.

Sapountzakis E.J and Dourakopoulos J.A. 2009. Nonlinear dynamic analysis of Timoshenko beams by BEM. Part II: Applications and Validation. Nonlinear Dynamics. vol. 58, No. 1, pp. 307-318.

Sathyamoorthy M. 1982a. Nonlinear analysis of beams, Part-I: A survey of recent advances. The Shock and Vibration Digest, vol. 14, pp. 19-35.

Sathyamoorthy M. 1982b. Nonlinear analysis of beams, Part-II: Finite-element methods. The Shock and Vibration Digest, vol. 14, pp. 7-18.

Srinivasan A.V. 1966. Nonlinear vibrations of beam and plates. International Journal of Nonlinear Mechanics, vol. 1, pp. 179191.

Wang R.T. and Chou T.H. 1998. Non-linear vibration of Timoshenko beam due to a moving force and the weight of beam. Journal of Sound and Vibration, vol. 218, No. 1, pp. 117-131.

Wu S.R. 2005. Classical solutions of forced vibration of rod and beam driven by displacement boundary conditions. Journal of Sound and Vibration, vol. 279, pp. 481-486.

\section{Biographical notes}

Anirban Mitra graduated from the Department of Mechanical Engineering, National Institute of Technology, Durgapur in the year 2006. Thereafter he completed his Master in Mechanical Engineering degree (Specialisation: Machine Design) from Jadavpur University in the year 2008. He is currently pursuing his PhD under the supervision of Prof. Kashinath Saha and Prof. Prasanta Sahoo in the Department of Mechanical Engineering, Jadavpur University. He is presently working as a senior research fellow (SRF) under the National Doctoral Fellowship (NDF) scheme of AICTE, India. His current area of research is large amplitude free and forced vibration analysis of stiffened plates in elastic and post-elastic regime.

Prasanta Sahoo is Professor at the Department of Mechanical Engineering, Jadavpur University, Kolkata, India. He received Bachelor of Mechanical Engineering from Jadavpur University and MTech and PhD in Mechanical Engineering from Indian Institute of Technology, Kharagpur, India. His research area is tribology and structural mechanics. He is the supervisor of $7 \mathrm{PhD}$ theses and 16 Master's theses. He has authored a textbook on Engineering Tribology (Prentice Hall of India, 2005) and book-chapters in Tribology Research Advances (Nova Science, 2009) and Tribology for Engineers - a practical guide (Woodhead Publishing, 2010). He has co-authored more than 150 technical papers in international journals and conference proceedings. He is on the editorial board of International Journal of Engineering, Science and Technology (Multicraft), Journal of Tribology Research (Serials), Journal of Tribology and Surface Engineering (Nova Science), International Journal of Manufacturing, Materials and Mechanical Engineering (IGI Global), Materials and Design (Elsevier). 
Kashinath Saha served Indian industry as design engineer in the sectors involving tea machineries, Industrial fan and air handling equipments and other heavy machinery components for five years. He joined academia as lecturer in the Department of Mechanical Engineering, Jadavpur University in 1987. He is pursuing teaching and research in the area of Machine Elements Design, Stress, deformation analysis and design of Structural Elements and Structural Stability Analysis. He has also carried out several experimental studies in the field of structural dynamics and supervised a number of post graduate thesis and research projects and has over 50 publications in international journals and conference proceedings.

Received December 2009

Accepted March 2010

Final acceptance in revised form May 2010 